\title{
Invariance Principles for Dependent Processes Indexed by Besov Classes with an Application to a Hausman Test for Linearity
}

\author{
Guido M. Kuersteiner* \\ University of Maryland
}

\begin{abstract}
This paper considers functional central limit theorems for stationary absolutely regular mixing processes. Bounds for the entropy with bracketing are derived using recent results in Nickl and Pötscher (2007). More specifically, their bracketing metric entropy bounds are extended to a norm defined in Doukhan, Massart and Rio (1995, henceforth DMR) that depends both on the marginal distribution of the process and on the mixing coefficients. Using these bounds, and based on a result in DMR, it is shown that for the class of weighted Besov spaces polynomially decaying tail behavior of the function class is sufficient to obtain a functional central limit theorem under minimal dependence conditions. A second class of functions that allow for a functional central limit theorem under minimal conditions are smooth functions defined on bounded sets. Similarly, a functional CLT for polynomially explosive tail behavior is obtained under additional moment conditions that are easy to check. An application to a Hausman (1978) specification test for linearity of the conditional mean illustrates the theory.
\end{abstract}

Keywords: dependent process, empirical process, mixing, Besov classes, Hausman test

\footnotetext{
*University of Maryland, Department of Economics, Tydings Hall 3145, 7343 Prinkert Dr., College Park, MD 20742, USA. email: kuersteiner@econ.umd.edu; http://econweb.umd.edu/ kuersteiner/

${ }^{\dagger}$ Very helpful comments received from the editor, Whitney Newey, and two anonymous referees are gratefully acknowledged.
} 


\section{Introduction}

This paper studies central limit theorems for empirical processes defined on dependent data and indexed by smooth classes of functions. Doukhan, Massart and Rio (1994) and Doukhan, Massart and Rio (1995) (henceforth DMR) are landmark contributions in this literature. The key insight from those papers is that a specific norm that combines dependence properties and the marginal distribution of the process provides the appropriate measure to assess the complexity of the function class in terms of bracketing entropy. However, as pointed out by Rio $(1998,2013)$ the results of DMR are not minimal in the sense of providing convergence under dependence assumptions equivalent to finite dimensional cases. In fact, for a $\beta$-mixing process with mixing coefficients $\beta_{m}$, central limit theorems can be established under the minimal condition that $\sum_{m=0}^{\infty} \beta_{m}<\infty$. Rio $(1998,2013)$ shows that such minimal results are possible in some cases involving Vapnik-Cervonenkis (VC) classes as well as certain Lipschitz type functions. In this paper the function classes for which such minimal results are possible are expanded to smooth classes of rapidly asymptoting functions as well as function classes defined on a bounded set. This is achieved by directly employing recent results of complexity measures for weighted Besov spaces in Haroske and Triebel (2005) and Nickl and Pötscher (2007). In addition to these improvements over the existing literature the paper also gives a number of explicit results that relate dependence properties of the underlying process to smoothness properties of the indexing function class.

Separate results then need to be employed to arrive at explicit central limit theorems. This is particularly relevant for dependent data where there is a potentially complex interaction between the properties of the function class, dependence of the process and properties of the marginal distribution of the process. An additional requirement, especially in econometric applications, is that function spaces are defined on unbounded sets, typically $\mathbb{R}^{d}$. This further limits applicability of many results available in the iid literature.

Andrews (1991) has given similar results under related conditions for processes that are not necessarily stationary. However, Andrews (1991) essentially is limited to function classes defined on a bounded domain. This paper compliments Andrews (1991) by allowing for weaker assumptions on the support of function classes while assuming stationarity and slightly stronger mixing conditions. Nickl (2007) mentions the possibility of obtaining explicit empirical process central limit theorems for the dependent case using the approach pursued here but does not give such results. A useful by-product of obtaining empirical central limit theorems for specific function classes are stochastic equicontinuity results for these function classes. This fact is exploited in the part of the paper that develops a 
Hausman specification test for the conditional mean function.

Empirical central limit theorems have a long history in probability and have found wide applications in statistics. Early results are due to Dudley $(1978,1984)$ and Pollard (1982). General results for iid data using bracketing were obtained by Ossiander (1987) and Pollard (1989). Results based on VC classes are due to Pollard (1990). Early results for dependent processes include Berkes and Phillip (1977) generalizing Donsker's theorem to strongly mixing stationary sequences. Uniform CLT's over function classes for dependent processes were studied in Doukhan, Leon and Portal (1987), Massart (1987), Andrews (1991), Andrews and Pollard (1994) and Hansen (1996). Arcones and Yu (1994) consider absolutely regular processes indexed by VC classes. A very influential paper is Doukhan, Massart and Rio (1995) which considers absolutely regular processes under a bracketing condition, extending results by Ossiander to the dependent case.

The paper is organized as follows. Section 2 presents definitions for measures of dependence and discusses the existing functional CLT's underlying the developments of the paper. Section 3 defines the smooth classes of function spaces considered. Section 4 presents the main theory and contains a detailed comparison with other related results in the literature. An application to the problem of testing the specification of the conditional mean using a Hausman test is presented in Section 5. Proofs are collected in the appendix in Section A,

\section{Notation and Existing Results}

The sequence $\chi_{t}$ consists of (measurable) random variables defined on the probability space $(\Omega, \mathcal{A}, \mathbb{P})$. Assume that $\left\{\chi_{t}\right\}_{t=-\infty}^{\infty}$ is strictly stationary with values in the measurable space $\left(\mathbb{R}^{d}, \mathcal{B}^{d}\right)$ where $\mathcal{B}^{d}$ is the Borel $\sigma$-field on $\mathbb{R}^{d}$ and $d \in \mathbb{N}_{+}$. Let $\mathcal{A}^{l}=\sigma\left(\chi_{t}: t \leq l\right)$ be the sigma field generated by $, \ldots \chi_{l-1}, \chi_{l}$ and similarly $\mathcal{D}^{l}=\sigma\left(\chi_{t}: t \geq l\right)$. Following DMR, p.379 the absolutely regular mixing coefficient $\beta_{m}$ is defined as

$$
2 \beta_{m}=\sup \sum_{(i, j) \in I \times J}\left|\mathbb{P}\left(A_{i} \cap D_{j}\right)-\mathbb{P}\left(A_{i}\right) \mathbb{P}\left(D_{j}\right)\right|
$$

where the supremum is taken over all finite partitions $A_{i}$ and $D_{j}$ of $\mathcal{A}^{0}$ and $\mathcal{D}^{m}$. The definition of $\beta_{m}$ is due to Volkonski and Rozanov (1959) who give an alternative equivalent formulation that is sometimes used in the literature (see for example Arcones and Yu, 1994). Strong mixing is defined as

$$
\alpha_{m}=\sup _{(A, D) \in \mathcal{A}^{0} \times \mathcal{D}^{m}}|\mathbb{P}(D \cap A)-\mathbb{P}(A) \mathbb{P}(D)|
$$


and $\varphi$-mixing is based on

$$
\varphi_{m}=\sup _{(A, D) \in \mathcal{A}^{0} \times \mathcal{D}^{m}}|\mathbb{P}(D \mid A)-\mathbb{P}(D)| .
$$

The relationship $2 \alpha_{m} \leq \beta_{m} \leq \varphi_{m} \leq 1$ holds. The condition

$$
\sum_{m=0}^{\infty} \beta_{m}<\infty
$$

is frequently imposed in what follows.

Define the Euclidian norm for a real valued matrix or vector $A$ as $\|A\|^{2}=\operatorname{tr} A A^{\prime}$. Let $\mathcal{X} \subseteq \mathbb{R}^{d}$ be a non-empty Borel set. Define the sup-norm $\|f\|_{\infty}=\sup _{x \in \mathcal{X}}|f(x)|$ for any measurable function $f: \mathcal{X} \rightarrow \mathbb{R}$. Similarly, for $r \geq 1$ let $\|f\|_{r, P}=\left(\int|f(x)|^{r} d P(x)\right)^{1 / r}$ where $P$ is the marginal distribution of $\chi_{t}$ and let $\mathcal{L}_{r}(P)$ be the set of functions with $\|f\|_{r, P}<\infty$. The following definitions are given in Rio (1993) and DMR. For a nonincreasing function $h: \mathbb{R} \rightarrow \mathbb{R}$ define the inverse $h^{-1}(u)=\inf \{t: h(t) \leq u\}$. Let $Q_{f}(u)$ be the quantile function defined as the inverse of the tail probability $P\left(\left|f\left(\chi_{t}\right)\right|>t\right)$. Let $\lfloor t\rfloor$ be the largest integer smaller or equal to $t \in \mathbb{R}$ and define $\beta^{-1}(u)=\inf \left\{t: \beta_{\lfloor t\rfloor} \leq u\right\}$. Now define the norm

$$
\|f\|_{2, \beta}=\sqrt{\int_{0}^{1} \beta^{-1}(u)\left(Q_{f}(u)\right)^{2} d u}<\infty .
$$

DMR, Lemma 1, show that if (11) holds, the set $\mathcal{L}_{2, \beta}(P)$ of functions with $\|f\|_{2, \beta}<\infty$ equipped with the norm $\|\cdot\|_{2, \beta}$ is a normed subspace of $\mathcal{L}_{2}(P)$ and that $\|f\|_{2, P} \leq\|f\|_{2, \beta}$. DMR (p.401) remark that $\sum_{m=0}^{\infty} \beta_{m}=\int_{0}^{1} \beta^{-1}(u) d u$. This implies that under the summability condition in (1) the space $\mathcal{L}_{2, \beta}(P)$ contains the space of bounded functions $\mathcal{L}_{\infty}(P)$. A reverse conclusion of their remark is that for bounded functions, $f \in \mathcal{L}_{2, \beta}(P)$ implies that $\sum_{m=0}^{\infty} \beta_{m}<\infty$ needs to hold.

Consider the class of functions $\mathcal{F}$ with elements $f: \mathcal{X} \rightarrow \mathbb{R}$. For a sample $\left\{\chi_{t}\right\}_{t=1}^{n}$ define the empirical process

$$
v_{n}(f)=n^{-1 / 2} \sum_{t=1}^{n}\left(f\left(\chi_{t}\right)-E\left[f\left(\chi_{t}\right)\right]\right) .
$$

When (11) is satisfied, Rio (1993, Theorem 1.2) shows that for $f \in \mathcal{L}_{2, \beta}(P)$,

$$
\sum_{t=-\infty}^{\infty}\left|\operatorname{Cov}\left(f\left(\chi_{0}\right), f\left(\chi_{t}\right)\right)\right| \leq 4\|f\|_{2, \beta}^{2}
$$

and for $\Gamma(f, f)=\sum_{t=-\infty}^{\infty} \operatorname{Cov}\left(f\left(\chi_{0}\right), f\left(\chi_{t}\right)\right)$ it follows that

$$
\lim _{n \rightarrow \infty} \operatorname{Var}\left(v_{n}(f)\right)=\Gamma(f, f) \leq 4\|f\|_{2, \beta}^{2} .
$$


Following DMR and van der Vaart and Wellner (1996, p.83) let $\mathcal{F}$ be a subset of a normed space $\left(V,\|\|_{V}\right)$ of functions $f: \mathcal{X} \rightarrow \mathbb{R}$ with norm \|\|$_{V}$. For any pair of functions, $l, u \in \mathcal{F}$ and $\delta>0$, the set $[l, u] \subset \mathcal{F}$ is a $\delta$-bracket if $l \leq u$ with $\|l-u\|_{V} \leq \delta$ and for all $f \in[l, u]$ it follows that $l \leq f \leq u$. The bracketing number $N_{[]}\left(\delta, \mathcal{F},\|\|_{V}\right)$ is the smallest number of $\delta$-brackets needed to cover $\mathcal{F}$. The entropy with bracketing is the logarithm of $N_{[]}\left(\delta, \mathcal{F},\|\|_{V}\right)$ denoted by $H_{[]}\left(\delta, \mathcal{F},\|\|_{V}\right)$.

DMR establish the following Theorem, see DMR, Theorem 1:

Theorem 1 (Doukhan, Massart and Rio, 1995) Assume that $\chi_{t}$ is a strictly stationary $\beta$-mixing sequence with (1) holding, marginal distribution $P$ and $\mathcal{F}$ a class of functions $f$ with $\mathcal{F} \subset \mathcal{L}_{2, \beta}(P)$ such that

$$
\int_{0}^{1} \sqrt{H_{[]}\left(\delta, \mathcal{F},\|\|_{2, \beta}\right)} d \delta<+\infty .
$$

Then the series $\sum_{t \in \mathbf{Z}} \operatorname{Cov}\left(f\left(\chi_{0}\right), f\left(\chi_{t}\right)\right)$ is absolutely convergent over $\mathcal{F}$ to a nonnegative quadratic form $\Gamma(f, f)$ and $(\Gamma(f, f))^{1 / 2}=\|f\|_{\Gamma} \leq 2\|f\|_{2, \beta}$. In addition there exists a sequence $\left(v^{(n)}\right)_{n>0}$ of Gaussian processes indexed by $\mathcal{F}$ with covariance function $\Gamma$ and a.s. uniformly continuous sample paths such that

$$
\sup _{f \in \mathcal{F}}\left|v_{n}(f)-v^{(n)}(f)\right| \rightarrow_{p} 0 \text { as } n \rightarrow \infty \text {. }
$$

The proof of Theorem 1 is given on p.409 of DMR and involves showing the convergence of the finite dimensional distributions as well as establishing a stochastic equicontinuity property. The finite dimensional vector $v_{n}\left(f_{1}\right), \ldots, v_{n}\left(f_{k}\right)$ converges weakly by a result of Doukhan, Massart and Rio (2014) such that

$$
\left(v_{n}\left(f_{1}\right), \ldots, v_{n}\left(f_{k}\right)\right) \rightarrow^{d}\left(v\left(f_{1}\right), \ldots, v\left(f_{k}\right)\right),
$$

where $v(f)$ is a Gaussian process with covariance function $\Gamma$ and a.s. uniformly continuous sample paths. The asymptotic equicontinuity condition is established by DMR (see p.410) and states that for every $\epsilon>0$ :

$$
\lim _{\delta \rightarrow 0} \limsup _{n \rightarrow \infty} \mathbb{P}^{*}\left(\sup _{\|f-g\|_{2, \beta} \leq \delta, f, g \in \mathcal{F}}\left|v_{n}(f)-v_{n}(g)\right|>\epsilon\right)=0,
$$

where $\mathbb{P}^{*}$ is outer probability. The short hand notation $v_{n}(f) \rightsquigarrow v(f)$ is used when both (3) and (4) hold. The implication of Theorem 1 that (4) holds as a sufficient condition for the statement of theorem is of independent interest in this paper and will be used in Section 5 for the analysis of semiparametric econometric procedures. 
Theorem 1 delivers a functional central limit theorem under close to minimal conditions on dependence and high level assumptions regarding the function classes it covers. Doukhan, Massart and Rio (1994) give a counter example where finite dimensional weak convergence fails for a function not in $\mathcal{L}_{2, \beta}(P)$. Rio (2013, p.104) notes that DMR's CLT holds for $\beta_{m}=O\left(m^{-b}\right)$ with $b>1$ and thus does not quite achieve minimal conditions on dependence. Rio $(1998,2013)$ provides functional CLT's for VC classes of functions as well as for classes of functions satisfying certain bracketing conditions under the minimal dependence assumption in (1).

The difficulties of obtaining results under minimal dependence assumptions as well as in applying the central limit theorem to particular statistical problems are related to verifying (2) for specific function classes. The bracketing integral convolutes conditions related to the dependence of the process, the marginal distribution of the process, tail behavior of the function class and smoothness restrictions of the function class into a single integrability condition. This paper extends results by Nickl and Pötscher (2007) on the bracketing properties of function spaces to disentangle these restrictions into conditions that can be individually verified in an application. In some cases this approach leads to functional CLT's under minimal dependence conditions.

In work preceding DMR, Ossiander (1987) obtains a version of Theorem 1 under independence. In that case, the bracketing integral is with respect to the $L_{2, P}$ norm $\|\cdot\|_{2, P}$. In applications one still needs to determine function classes that satisfy (2). Specific results for this case were obtained by Nickl and Pötscher (2007) who also provide references to the previous literature.

\section{Function Spaces}

The purpose of this section is to introduce the function spaces for which the bracketing condition in Theorem 1 is verified. The most general class of function spaces considered are Besov spaces. Of particular importance are weighted Besov spaces which provide a mechanism to handle functions with unbounded support that do not vanish in the tails. Special cases of Besov spaces such as Sobolev, Hölder and Zygmund spaces are introduced subsequently.

The definition of Besov spaces follows Nickl and Pötscher (2007, Remark 2). For Lebesgue measure $\lambda$ let $\mathcal{L}_{p}\left(\mathbb{R}^{d}, \lambda\right)$ be the set of all functions $f: \mathbb{R}^{d} \rightarrow \mathbb{R}$ with $\|f\|_{p, \lambda}=$ $\left(\int|f(x)|^{p} d x\right)^{1 / p}<\infty$. Let $\alpha=\left(\alpha_{1}, \ldots, \alpha_{d}\right)$ be a multi index of non-negative integers $\alpha_{i}$ with $|\alpha|=\sum_{i=1}^{d} \alpha_{i}$ and let $D^{\alpha}$ denote the partial differential operator $\partial^{|\alpha|} /\left(\left(\partial x_{1}\right)^{\alpha_{1}} \ldots\left(\partial x_{d}\right)^{\alpha_{d}}\right)$ 
of order $|\alpha|$ in the sense of distributions - see Stein (1970, p. 121). For a function $f: \mathbb{R}^{d} \rightarrow \mathbb{R}$ the difference operator $\Delta_{z}$ is defined as $\Delta_{z} f()=.f(.+z)-f($.$) and \Delta_{z}^{2} f()=.\Delta_{z}\left(\Delta_{z} f().\right)$ for $z \in \mathbb{R}^{d}$. Let $0<s<\infty$ and set $s=[s]^{-}+\{s\}^{+}$where $[s]^{-}$is integer and $0<\{s\}^{+} \leq 1$. For example, when $s=1,\{s\}^{+}=1$ and $[s]^{-}=0$. Let $1 \leq p \leq \infty$ and $1 \leq q \leq \infty$. For $f \in$ $\mathcal{L}_{p}\left(\mathbb{R}^{d}, \lambda\right)$ with $\left\|D^{\alpha} f\right\|_{p, \lambda}<\infty$ and for $0 \leq \alpha \leq[s]^{-}$define

$$
\|f\|_{s, p, q, \lambda}^{*}=\sum_{0 \leq \alpha \leq[s]^{-}}\left\|D^{\alpha} f\right\|_{p, \lambda}+\sum_{\alpha=[s]^{-}}\left(\int_{\mathbb{R}^{d}}|z|^{-\{s\}^{+} q-d}\left\|\Delta_{z}^{2} D^{\alpha} f\right\|_{p, \lambda}^{q} d z\right)^{1 / q}
$$

for $q<\infty$, and for $q=\infty$ define

$$
\|f\|_{s, p, \infty, \lambda}^{*}=\sum_{0 \leq \alpha \leq[s]^{-}}\left\|D^{\alpha} f\right\|_{p, \lambda}+\sum_{\alpha=[s]^{-}} \sup _{0 \neq z \in \mathbb{R}^{d}}|z|^{-\{s\}^{+}}\left\|\Delta_{z}^{2} D^{\alpha} f\right\|_{p, \lambda} .
$$

The Besov space $\mathcal{B}_{p q}^{s}\left(\mathbb{R}^{d}\right)$ is defined as $\mathcal{B}_{p q}^{s}\left(\mathbb{R}^{d}\right)=\left\{f \in \mathcal{L}_{p}\left(\mathbb{R}^{d}, \lambda\right):\|f\|_{s, p, q, \lambda}^{*}<\infty\right\}$. An equivalent definition can be given in terms of Fourier transforms $F$ acting on the space of complex tempered distributions on $\mathbb{R}^{d}$ (see Edmunds and Triebel, 1996, 2.2.1). Denote by $F^{-1}$ the inverse of $F$. Let $\varphi_{0}(x)$ be a complex valued $C^{\infty}$-function on $\mathbb{R}^{d}$ with $\varphi_{0}(x)=1$ if $\|x\| \leq 1$ and $\varphi_{0}(x)=0$ if $\|x\| \geq 3 / 2$. Define $\varphi_{1}(x)=\varphi_{0}(x / 2)-\varphi_{0}(x)$ and $\varphi_{k}(x)=$ $\varphi_{1}\left(2^{-k+1} x\right)$ for $k \in \mathbb{N}$. Let $0 \leq s<\infty, 1 \leq p \leq \infty, 1 \leq q \leq \infty$, with $q=1$ if $s=0$. For $f \in \mathcal{L}_{p}\left(\mathbb{R}^{d}, \lambda\right)$ and $q<\infty$ define

$$
\|f\|_{s, p, q, \lambda}=\left(\sum_{k=0}^{\infty} 2^{k s q}\left\|F^{-1}\left(\varphi_{k} F f\right)\right\|_{p, \lambda}^{q}\right)^{1 / q}
$$

and for $q=\infty$

$$
\|f\|_{s, p, \infty, \lambda}=\sup _{0 \leq k<\infty} 2^{k s}\left\|F^{-1}\left(\varphi_{k} F f\right)\right\|_{p, \lambda} .
$$

Then, it follows (see Nickl and Pötscher, 2007, p. 180) that

$$
\mathcal{B}_{p q}^{s}\left(\mathbb{R}^{d}\right)=\left\{f \in \mathcal{L}_{p}\left(\mathbb{R}^{d}, \lambda\right):\|f\|_{s, p, q, \lambda}<\infty\right\}
$$

and the norms $\|f\|_{s, p, q, \lambda}^{*}$ and $\|f\|_{s, p, q, \lambda}$ are equivalent on $\mathcal{B}_{p q}^{s}\left(\mathbb{R}^{d}\right)$. Define $\langle x\rangle=1+\|x\|^{2}$. Weighted Besov spaces are now defined as in Edmunds and Triebel $(1996,4.2)$ and Nickl and Pötscher (2007, p.181) for $\vartheta \in \mathbb{R}$ as

$$
\mathcal{B}_{p q}^{s}\left(\mathbb{R}^{d}, \vartheta\right)=\left\{f:\left\|f(.)\langle x\rangle^{\vartheta / 2}\right\|_{s, p, q, \lambda}<\infty\right\} .
$$

For $s>d / p$ or $s=d / p$ with $q=1$ define

$$
B_{p q}^{s}\left(\mathbb{R}^{d}, \vartheta\right)=\mathcal{B}_{p q}^{s}\left(\mathbb{R}^{d}, \vartheta\right) \cap\left\{f: f(.)\langle x\rangle^{\vartheta / 2} \in C\left(\mathbb{R}^{d}\right)\right\}
$$


where $C\left(\mathbb{R}^{d}\right)$ is the vector space of bounded continuous real valued functions on $\mathbb{R}^{d}$ with the sup-norm $\|\cdot\|_{\infty}$. Nickl and Pötscher (2007, Proposition 3) show that $f \in B_{p q}^{s}\left(\mathbb{R}^{d}\right)$ implies that $f$ is bounded and if $p<\infty$ it also follows that $\lim _{\|x\| \rightarrow \infty} f(x)=0$. These restrictions do not necessarily apply when $f \in B_{p q}^{s}\left(\mathbb{R}^{d}, \vartheta\right)$ and $\vartheta<0$. This feature of weighted spaces is important for applications in econometrics, as will be demonstrated in Section 5 .

A special case of Besov spaces are Sobolev spaces. They are defined as follows (see Nickl and Pötscher, 2007, Section 3.3.2). Let $1<p<\infty$, real $s \geq 0$ and

$$
\mathcal{H}_{p}^{s}\left(\mathbb{R}^{d}\right)=\left\{f \in \mathcal{L}_{p}\left(\mathbb{R}^{d}, \lambda\right):\|f\|_{s, p, \lambda} \equiv\left\|F^{-1}\left(\langle x\rangle^{s} F f\right)\right\|_{p, \lambda}<\infty\right\}
$$

where the norms are formulated in terms of the Fourier transform $F$. When $s \geq 0$ is integer, an equivalent (semi)norm on $\mathcal{H}_{p}^{s}\left(\mathbb{R}^{d}\right)$ is given by

$$
\|f\|=\sum_{0 \leq|\alpha| \leq s}\left\|D^{\alpha} f\right\|_{p, \lambda} .
$$

Similar as before define the Banach space $H_{p}^{s}\left(\mathbb{R}^{d}\right)$ of continuous functions for $s>d / p$ as

$$
H_{p}^{s}\left(\mathbb{R}^{d}\right)=\mathcal{H}_{p}^{s}\left(\mathbb{R}^{d}\right) \cap\left\{f: \in C\left(\mathbb{R}^{d}\right)\right\} .
$$

The weighted Sobolev space is given by

$$
H_{p}^{s}\left(\mathbb{R}^{d}, \vartheta\right)=\left\{f: f(.)\langle x\rangle^{\vartheta / 2} \in H_{p}^{s}\left(\mathbb{R}^{d}\right)\right\} .
$$

For $s>0, s$ not integer, the Hölder space is defined as the space $C^{s}\left(\mathbb{R}^{d}\right)$ of all $\lfloor s\rfloor$-times differentiable functions $f$ with finite norm

$$
\|f\|_{s, \infty}=\sum_{0 \leq|\alpha| \leq\lfloor s\rfloor}\left\|D^{\alpha} f\right\|_{\infty}+\sum_{|\alpha|=\lfloor s\rfloor} \sup _{x \neq y} \frac{\left|D^{\alpha} f(x)-D^{\alpha} f(y)\right|}{|x-y|^{s-\lfloor s\rfloor}} .
$$

The weighted space $C^{s}\left(\mathbb{R}^{d}, \vartheta\right)$ is given by

$$
C^{s}\left(\mathbb{R}^{d}, \vartheta\right)=\left\{f:\left\|f(.)\langle x\rangle^{\vartheta / 2}\right\|_{s, \infty}<\infty\right\} .
$$

Related is the Zygmund space $\mathcal{C}^{s}\left(\mathbb{R}^{d}\right)$ for $s>0$ defined in Triebel (1983, p.36) or Triebel (1992, p.4). Let

$$
\|f\|_{s, \infty}^{z}=\sum_{0 \leq|\alpha| \leq[s]^{-}}\left\|D^{\alpha} f\right\|_{\infty}+\sum_{|\alpha|=[s]^{-}} \sup _{0 \neq z \in \mathbb{R}^{d}}|z|^{-\{s\}^{+}}\left\|\Delta_{z}^{2} D^{\alpha} f\right\|_{\infty} .
$$

By Triebel $\left(1992\right.$, p.5), $\mathcal{C}^{s}\left(\mathbb{R}^{d}, \vartheta\right)=C^{s}\left(\mathbb{R}^{d}, \vartheta\right)$ when $s>0$ and $s$ is not integer.

Let $\mathfrak{X} \subset \mathbb{R}^{d}$ be a bounded Borel set. The space $C^{s}(\mathfrak{X})$ is considered by van der Vaart and Wellner (1996, p. 154) under the additional constraint that $\|f\|_{s, \infty} \leq M$ for some bounded constant $M$. As noted there, when $0<s<1, C^{s}(\mathfrak{X})$ contains the Lipschitz functions (see Adams and Fournier 2003, Theorem 1.34). 


\section{New Results}

The following result gives upper bounds for entropy with bracketing on the normed space $\mathcal{L}_{2, \beta}(P)$. It extends Theorem 1 of Nickl and Pötscher $(2007)$ to the space $\mathcal{L}_{2, \beta}(P)$ which plays a crucial role in obtaining a functional CLT for dependent processes.

Theorem 2 Assume that $1 \leq p \leq \infty, 1 \leq q \leq \infty, \vartheta \in \mathbb{R}$ and $s>d / p$. Further assume that $\mathcal{F} \subset B_{p q}^{s}\left(\mathbb{R}^{d}, \vartheta\right)$ is nonempty and bounded. If $\vartheta>0$ then

$$
H_{[]}\left(\delta, \mathcal{F},\|\|_{2, \beta}\right) \precsim\left\{\begin{array}{cc}
\delta^{-d / s} & \text { if } \vartheta>s-d / p \\
\delta^{-(\vartheta / d+1 / p)^{-1}} & \text { if } \vartheta<s-d / p
\end{array} .\right.
$$

If $\vartheta \leq 0$ and if for some $\gamma>0$ it holds that

$$
\left\|\left\langle\chi_{t}\right\rangle^{(\gamma-\vartheta) / 2}\right\|_{2, \beta}<\infty
$$

then it follows that

$$
H_{[]}\left(\delta, \mathcal{F},\|\|_{2, \beta}\right) \precsim\left\{\begin{array}{cc}
\delta^{-d / s} & \text { if } \gamma>s-d / p \\
\delta^{-(\gamma / d+1 / p)^{-1}} & \text { if } \gamma<s-d / p
\end{array} .\right.
$$

The difference between Nickl and Pötscher (2007, Theorem 1) and Theorem 2 is that bracketing is with respect to the norm $\|\cdot\|_{2, \beta}$ rather than the conventional $\|\cdot\|_{r, P}$ norm on $\mathcal{L}_{r}\left(\mathbb{R}^{d}, P\right)$. Theorem 2 directly leads to a functional CLT based on the theory of DMR. A corollary to Theorem 2 is obtained for the case when the function space $\mathcal{F}$ is restricted to a bounded domain $\mathfrak{X}$.

Corollary 3 Let $\mathfrak{X} \subset \mathbb{R}^{d}$ and there exists a finite $M$ with $\langle x\rangle \leq M$ for all $x \in \mathfrak{X}$. Assume that $1 \leq p \leq \infty, 1 \leq q \leq \infty, \vartheta \in \mathbb{R}$ and $s>d / p$. Further assume that $\mathcal{F} \subset B_{p q}^{s}(\mathfrak{X}, \vartheta)$ is nonempty and bounded. Then,

$$
H_{[]}\left(\delta, \mathcal{F},\|\|_{2, \beta}\right) \precsim\left\{\begin{array}{cl}
\delta^{-d / s} & \text { if } \vartheta>s-d / p \\
\delta^{-(\vartheta / d+1 / p)^{-1}} & \text { if } \vartheta<s-d / p
\end{array} .\right.
$$

The bounds on bracketing numbers obtained in Theorem 2 and Corollary 3 can now be applied to obtain a functional central limit theorem based on Theorem 1 of DMR. The proof uses the tail decay properties of weighted function spaces to establish that $\mathcal{F} \subset \mathcal{L}_{2, \beta}(P)$. This property is satisfied without further assumptions about the marginal distribution of $\chi_{t}$ if $\vartheta>0$. 
Theorem 4 Let $\chi_{t}$ be a strictly stationary and $\beta$-mixing process. Assume that (1) holds. Assume that $1 \leq p \leq \infty, 1 \leq q \leq \infty, \vartheta \in \mathbb{R}$ and $s>d / p$. Further assume that $\mathcal{F} \subset B_{p q}^{s}\left(\mathbb{R}^{d}, \vartheta\right)$ is nonempty and bounded. Assume that one of the following conditions hold:

(i) $\vartheta>0, \vartheta>s-d / p$ and $s / d>1 / 2$;

(ii) $\vartheta>0, \vartheta<s-d / p$ and $\vartheta / d+1 / p>1 / 2$;

(iii) $\vartheta \leq 0$ and for some $\gamma>0$ it follows that $\left\|\left\langle\chi_{t}\right\rangle^{(\gamma-\vartheta) / 2}\right\|_{2, \beta}<\infty, \gamma>s-d / p$ and $s / d>1 / 2$

(iv) $\vartheta \leq 0$ and for some $\gamma>0$ it follows that $\left\|\left\langle\chi_{t}\right\rangle^{(\gamma-\vartheta) / 2}\right\|_{2, \beta}<\infty, \gamma<s-d / p$ and $\gamma / d+1 / p>1 / 2$.

Then, $v_{n}(f) \rightsquigarrow v(f)$ where $v(f)$ is a Gaussian process with covariance function $\Gamma$ and a.s. uniformly continuous sample paths.

Note that the conditions $1 / 2<s / d$ and $1 / 2<\gamma / d+1 / p$ are the same as the conditions given in Corollary 5 of Nickl and Pötscher (2007) for the iid case. In the time series case these conditions need to hold in conjunction with bounds on the $\beta$-mixing coefficients and, when $\vartheta \leq 0$, the moment condition $\left\|\left\langle\chi_{t}\right\rangle^{(\gamma-\vartheta) / 2}\right\|_{2, \beta}<\infty$.

Theorem 4 shows that an empirical process CLT can be obtained under the minimal Condition (11) if $\mathcal{F}$ is a space of functions that asymptote to zero rapidly enough, measured by the parameter $\vartheta>0$. If the decay is rapid enough relative to smoothness as in case (i) then the functional CLT holds under the minimal condition $s / d>1 / 2$. Even in case (ii) one still obtains a result with only Condition (10) imposed on the dependence of the process.

When $\vartheta \leq 0$ the CLT only holds under additional moment restrictions and summability conditions for the $\beta$-mixing coefficients that are stronger than those imposed by (11). The $\|\cdot\|_{2, \beta}$ norm provides a compact summary of these conditions at the cost of being less easy to apply to statistical problems. It is also harder to compare results formulated for bounds on $\|\cdot\|_{2, \beta}$ with results in the literature. Theorem [6 below gives sufficient conditions in terms of moments for $\chi_{t}$ and the summability of mixing coefficients without directly relying on the $\|\cdot\|_{2, \beta}$ norm.

The results given here complement the ones in Rio (2013). If a process is strictly stationary and $\beta$-mixing with Condition (10) and $f \in B_{p q}^{s}\left(\mathbb{R}^{d}, \vartheta\right)$ with $\vartheta>s-d / p$ then Theorem 4 (i) establishes a functional CLT under the conditions that $s>d / p$ and $s / d>1 / 2$. In particular, if $p=\infty$, then the FCLT holds under the minimal condition that $\vartheta>s>0$ and $s / d>1 / 2$. This case is not covered by the results in Rio (2013). To see this note that $B_{p_{1} \infty}^{s}\left(\mathbb{R}^{d}\right) \subset B_{p_{2} \infty}^{s+d / p_{1}-d / p_{2}}\left(\mathbb{R}^{d}\right)$ for $p_{1} \leq p_{2} \leq \infty$ by Triebel $(1983,2.7 .1)$ indicating that 
the class $B_{p \infty}^{s}\left(\mathbb{R}^{d}\right)$ for $p>2$, which is covered by Theorem 4 , is a larger class than the one considered by Rio (2013). Further, from Haroske and Triebel (1994, 2005) it follows for $\vartheta>0, \vartheta / d<1, s_{1}-s_{2}>0$ and $p_{1}(1-\vartheta / d)<p_{2}$ that $B_{p_{1} \infty}^{s_{1}}\left(\mathbb{R}^{d}, \vartheta\right)$ is embedded in $B_{p_{2} \infty}^{s_{2}}\left(\mathbb{R}^{d}\right)$. For example, when $d=1$ the constraints $s>1 / 2, s>1 / p, \vartheta<1$ and $p_{1}(1-\vartheta)<2$ must hold for $B_{p_{1} \infty}^{s_{1}}\left(\mathbb{R}^{d}, \vartheta\right)$ to be embedded in $B_{p_{2} \infty}^{s_{2}}\left(\mathbb{R}^{d}\right)$. Thus, for Rio's results to encompass Theorem 4 one needs $p<2 /(1-\vartheta)$. The results of Rio (2013) then cover the spaces $B_{p \infty}^{s}\left(\mathbb{R}^{d}, \vartheta\right)$ for values of $\vartheta<1$ and values of $p \leq \infty$. However, as $\vartheta$ approaches 0 , the largest value $p$ can take approaches 2 while such a constraint does not apply to Theorem 4. On the other hand, Rio (2013) covers cases with $\vartheta=0$ and $p \leq 2$ which can only be handled by Theorem 4 under additional moment restrictions and stronger assumptions on the $\beta$-mixing coefficients.

When $p=2$, then $s / d>1 / 2$ and $\vartheta>s-d / 2$ lead to a FCLT by means of Theorem 4. This case essentially corresponds to Rio (2013) when $s-d / 2$ is close to zero. By Triebel $(1983,2.7 .1)$ it follows that $B_{p q}^{s_{1}}\left(\mathbb{R}^{d}, \vartheta\right)$ is continuously embedded in $B_{p q}^{s_{0}}\left(\mathbb{R}^{d}, \vartheta\right)$ for $s_{1} \geq s_{0}$. Thus, to apply Theorem 4 one can always choose $s$ small enough such that $s-d / 2$ is arbitrarily small and therefore $\vartheta$ can be chosen small. If $\vartheta<s-d / p$ then Theorem 4 (ii) holds under the condition that $\vartheta / d>1 / 2+1 / p$ such that the CLT holds for $p$ sufficiently large and $s / d>1 / 2$.

These arguments indicate that the results in Rio (2013) are slightly sharper for the case when $p \in[1,2]$ because of the requirement in Theorem 4 that $\vartheta>s-d / p$. In addition, by Triebel $(1983,2.3 .2$, Proposition 2$), B_{p q_{0}}^{s}\left(\mathbb{R}^{d}, \vartheta\right)$ is continuously embedded in $B_{p q_{1}}^{s}\left(\mathbb{R}^{d}, \vartheta\right)$ for $q_{0} \leq q_{1} \leq \infty$ and $p>0$ such that $B_{p q}^{s}\left(\mathbb{R}^{d}\right)$ is continuously embedded in $\operatorname{Lip}^{*}\left(s, p, \mathbb{R}^{d}\right)$. This implies that the results in Rio cover the spaces $B_{p q}^{s}\left(\mathbb{R}^{d}\right)$ for $p \in[1,2]$ and $q \leq \infty$.

In summary, the results in Theorem 4 are very similar to Rio (2013) when $p \leq 2$ and the tail behavior of the function class is controlled by a polynomial. However, the results are achieved with simpler proofs. Because of the embedding result in Triebel (1983, 2.7.1), additional function classes are covered by Theorem 4 that are not contained in Rio (2013) when $p>2$. Theorem 4 also covers cases when $\vartheta \leq 0$ and $p \leq \infty$ that are not covered by Rio (2013). However, in these situations somewhat stronger assumptions than (11) need to be imposed on dependence. Here the case $\vartheta=0$ and $p=\infty$ may be of particular interest since the tail behavior of $f(x)$ no longer necessarily satisfies $\lim _{\|x\|} f(x) \rightarrow 0$ (see Proposition 3 of NP). This is one example of a case not covered by the results in Rio (2013).

Another result that is not directly covered by Theorem《is Rio (2013, Theorem 8.1). Rio considers the generalized Lipschitz spaces $\operatorname{Lip}^{*}\left(s, p, \mathbb{R}^{d}\right)$ defined in Meyer (1992). Meyer 
(1992, Proposition 7, p. 200) shows that every $f \in \operatorname{Lip}^{*}\left(s, p, \mathbb{R}^{d}\right)$ is in $\mathcal{B}_{p \infty}^{s}\left(\mathbb{R}^{d}\right)$. Rio (2013, Proposition 8.1) gives an equivalent norm $\|f\|_{\text {ond }}$ for functions $f \in \operatorname{Lip}^{*}\left(s, p, \mathbb{R}^{d}\right)$. Rio shows that for every strongly mixing and stationary sequence with $\sum_{m=1}^{\infty} \alpha_{m}<\infty$, $f \in \operatorname{Lip}^{*}\left(s, p, \mathbb{R}^{d}\right)$ with $p \in[1,2], s>d / p$ and $\|f\|_{\text {ond }} \leq a$ for some constant $a<\infty$, the empirical process $v_{n}(f)$ satisfies a stochastic equicontinuity condition and thus a functional central limit theorem. Rio (2013, Theorem 8.1) is not covered by the theory in this paper because the concept of strongly mixing sequences is slightly weaker than $\beta$-mixing.

An immediate corollary to Theorem 4 obtains for the case where $\chi_{t}$ takes values in a bounded set $\mathfrak{X} \subset \mathbb{R}^{d}$.

Corollary 5 Let $\chi_{t}$ be strictly stationary and $\beta$-mixing. Assume that $P\left(\chi_{t} \in \mathfrak{X}\right)=1$ for a bounded Borel set $\mathfrak{X} \subset \mathbb{R}^{d}$ and there exists a finite $M$ with $\langle x\rangle \leq M$ for all $x \in \mathfrak{X}$. Assume that (1) holds. Assume that $1 \leq p \leq \infty, 1 \leq q \leq \infty, \vartheta \in \mathbb{R}$ and for $s, d<\infty$ and $s>d / p$. Further assume that $\mathcal{F} \subset B_{p q}^{s}(\mathfrak{X})$ is nonempty and bounded. Assume that $s / d>1 / 2$. Then, $v_{n}(f) \rightsquigarrow v(f)$ where $v(f)$ is a Gaussian process with covariance function $\Gamma$ and a.s. uniformly continuous sample paths.

Corollary 5 show that for smooth function classes restricted to a bounded set a functional CLT holds under the minimal dependence condition (11).

When the asymptotic behavior of $f$ as $\|x\| \rightarrow \infty$ is proportional to $\left\langle\chi_{t}\right\rangle^{-\vartheta / 2}$ and $\vartheta \leq 0$, then more restrictive conditions on the dependence need to be imposed. This happens implicitly through the condition

$$
\left\|\left\langle\chi_{t}\right\rangle^{(\gamma-\vartheta) / 2}\right\|_{2, \beta}<\infty
$$

which must hold for some $\gamma>0$. The advantage of this condition is that it only involves the marginal distribution of $\chi_{t}$ and not the properties of the functional class, other than through the parameter $\vartheta$. Results in DMR can be used to give simple sufficient conditions for 5. Under additional assumptions about the order of $\beta_{m}$ and moment restrictions on the marginal distribution of $\left\|\chi_{t}\right\|^{2}$ the following result can be given for the case when $\vartheta \leq 0$, i.e. when $\lim _{\|x\|} f(x) \rightarrow 0$ does not necessarily hold.

Theorem 6 Let $\chi_{t}$ be strictly stationary and $\beta$-mixing. Assume that for some $r>1$, $\sum_{m=1}^{\infty} m^{1 /(r-1)} \beta_{m}<\infty$ holds. Assume that $1 \leq p \leq \infty, 1 \leq q \leq \infty, \vartheta \in \mathbb{R}, \vartheta \leq 0$ and $s>d / p$. Further assume that $\mathcal{F} \subset B_{p q}^{s}\left(\mathbb{R}^{d}, \vartheta\right)$ is nonempty and bounded. Assume that for some $\gamma>0$ such that $r(\gamma-\vartheta)>1$ it holds that $E\left[\left\|\chi_{t}\right\|^{2 r(\gamma-\vartheta)}\right]<\infty$ and that either (i) $\gamma>s-d / p$ and $s / d>1 / 2$ or 
(ii) $\gamma<s-d / p$ and $\gamma / d+1 / p>1 / 2$.

Then, $v_{n}(f) \rightsquigarrow v(f)$ where $v(f)$ is a Gaussian process with covariance function $\Gamma$ and a.s. uniformly continuous sample paths.

The form of the last theorem is particularly useful when a comparison with other results in the literature is desired, since those results are often presented in terms of separate moment bounds and size restrictions on mixing coefficients.

More generally, the results show that in weighted Besov spaces control over tail behavior of the function class can be utilized to give sufficient conditions for a CLT that directly involves the marginal distribution of $\chi_{t}$ rather than that of $f\left(\chi_{t}\right)$. This is possible because the asymptotic behavior of $f\left(\chi_{t}\right)$ is controlled by terms that are functions of $\left\|\chi_{t}\right\|$. The next corollary gives explicit versions of the previous general results for Sobolev, Hölder and Lipschitz classes of functions.

The following Corollary is a special case of Theorem 4. The proof follows in the same way as the proofs of similar corollaries in Nickl and Pötscher (2007) by arguing that bounded subsets of $H_{p}^{s}\left(\mathbb{R}^{d}, \vartheta\right)$ are also bounded subsets of $B_{p \infty}^{s}\left(\mathbb{R}^{d}, \vartheta\right)$.

Corollary 7 Let $\chi_{t}$ be a strictly stationary and $\beta$-mixing process. Assume that (1) holds. Assume that $1<p \leq \infty, \vartheta \in \mathbb{R}$ and $s>d / p$. Further assume that $\mathcal{F} \subset H_{p}^{s}\left(\mathbb{R}^{d}, \vartheta\right)$ is nonempty and bounded. Assume that one of the following conditions hold:

(i) $\vartheta>0, \vartheta>s-d / p$ and $s / d>1 / 2$;

(ii) $\vartheta>0, \vartheta<s-d / p$ and $\vartheta / d+1 / p>1 / 2$;

(iii) $\vartheta \leq 0$ and for some $\gamma>0$ it follows that $\left\|\left\langle\chi_{t}\right\rangle^{(\gamma-\vartheta) / 2}\right\|_{2, \beta}<\infty, \gamma>s-d / p$ and $s / d>1 / 2$

(iv) $\vartheta \leq 0$ and for some $\gamma>0$ it follows that $\left\|\left\langle\chi_{t}\right\rangle^{(\gamma-\vartheta) / 2}\right\|_{2, \beta}<\infty, \gamma<s-d / p$ and $\gamma / d+1 / p>1 / 2$.

Then, $v_{n}(f) \rightsquigarrow v(f)$ where $v(f)$ is a Gaussian process with covariance function $\Gamma$ and a.s. uniformly continuous sample paths.

The following corollary again considers the special case where the domain of the function space is a bounded subset of $\mathbb{R}^{d}$.

Corollary 8 Let $\chi_{t}$ be a strictly stationary and $\beta$-mixing process. Assume that $P\left(\chi_{t} \in \mathfrak{X}\right)=$ 1 where $\mathfrak{X} \subset \mathbb{R}^{d}$ and there exists a finite $M$ with $\langle x\rangle \leq M$ for all $x \in \mathfrak{X}$. Assume that (1) holds. Assume that $1<p \leq \infty, \vartheta \in \mathbb{R}$ and $s, d<\infty$ with $s>d / p$. Further assume that $\mathcal{F} \subset H_{p}^{s}(\mathfrak{X}, \vartheta)$ is nonempty and bounded. Assume that $s / d>1 / 2$. Then, $v_{n}(f) \rightsquigarrow v(f)$ 
where $v(f)$ is a Gaussian process with covariance function $\Gamma$ and a.s. uniformly continuous sample paths.

Andrews (1991) considers the space $\mathcal{H}_{p}^{s}(\mathfrak{X})$ where $\mathfrak{X}$ is a bounded subset of $\mathbb{R}^{d}$. He allows for heterogeneous near epoch dependent processes which include as special cases strong mixing stationary sequences. Since $\beta$-mixing implies strong mixing the results of this paper are obtained under somewhat stronger assumptions as far as the mixing concept and stationarity requirements are concerned. On the other hand, no boundedness of $\mathfrak{X}$ is required. Andrews (1991, p.199) discusses some ways of relaxing the boundedness assumption regarding the support but does not provide a general treatment. Moreover, as pointed out by Nickl and Pötscher (2007, p. 179 and p. 196) it follows for $f \in H_{p}^{s}\left(\mathbb{R}^{d}\right)$, $\lim _{\|x\|} f(x) \rightarrow 0$ while this is not necessarily the case for $f \in H_{p}^{s}\left(\mathbb{R}^{d}, \vartheta\right)$ and $\vartheta<0$.

Andrews (1991, Theorem 4 and Comment 1) obtains a functional central limit theorem for strong mixing processes of size $-2, f \in \mathcal{H}_{2}^{s}(\mathfrak{X})$ and $s / d>1 / 2$. Corollary 8 shows that, at least under the additional assumption of stationarity and $\beta$-mixing but only satisfying (11), this result can be obtained for all functions in $H_{p}^{s}(\mathfrak{X})$ with $s / d>1 / 2$. Note that a $\beta$ mixing process that satisfies Condition (11) also is $\alpha$-mixing with $\sum_{m=1}^{\infty} \alpha_{m}<\infty$ but is not necessarily $\alpha$-mixing of size -2 . In this sense, the conditions given here are complementary to Andrews (1991).

The following corollaries specialize previous results to Hölder spaces.

Corollary 9 Let $\chi_{t}$ be strictly stationary and $\beta$-mixing. Assume that (1) holds. Assume that $\vartheta \in \mathbb{R}$ and $s>d / 2$. Further assume that $\mathcal{F} \subset C^{s}\left(\mathbb{R}^{d}, \vartheta\right)$ is nonempty and bounded. Assume that one of the following conditions hold:

(i) $\vartheta>0, \vartheta>s$ and $s / d>1 / 2$;

(ii) $\vartheta>0, \vartheta<s$ and $\vartheta / d>1 / 2$

(iii) $\vartheta \leq 0$ and for some $\gamma>0$ it follows that $\left\|\left\langle\chi_{t}\right\rangle^{(\gamma-\vartheta) / 2}\right\|_{2, \beta}<\infty, \gamma>s$ and $s / d>1 / 2$;

(iv) $\vartheta \leq 0$ and for some $\gamma>0$ it follows that $\left\|\left\langle\chi_{t}\right\rangle^{(\gamma-\vartheta) / 2}\right\|_{2, \beta}<\infty, \gamma<s$ and $\gamma / d>1 / 2$. Then, $v_{n}(f) \rightsquigarrow v(f)$ where $v(f)$ is a Gaussian process with covariance function $\Gamma$ and a.s. uniformly continuous sample paths.

The proof follows again from noting that $\mathcal{F}$ is a bounded subset in $B_{\infty \infty}^{s}\left(\mathbb{R}^{d}, \vartheta\right)$, see Nickl and Pötscher (2007, p. 188). As before, additional results for the cases of bounded support can be stated as follows. 
Corollary 10 Let $\chi_{t}$ be a strictly stationary and $\beta$-mixing. Assume that $P\left(\chi_{t} \in \mathfrak{X}\right)=1$ where $\mathfrak{X} \subset \mathbb{R}^{d}$ and there exists a finite $M$ with $\langle x\rangle \leq M$ for all $x \in \mathfrak{X}$. Assume that (1) holds. Assume that $\vartheta \in \mathbb{R}, s, d<\infty$ and $s>0$. Further assume that $\mathcal{F} \subset C^{s}(\mathfrak{X}, \vartheta)$ is nonempty and bounded. Assume that $s / d>1 / 2$. Then, $v_{n}(f) \rightsquigarrow v(f)$ where $v(f)$ is a Gaussian process with covariance function $\Gamma$ and a.s. uniformly continuous sample paths.

Andrews (1991, Comment 3) also considers the case of strong mixing processes of size -2 and Lipschitz function classes. More specifically, when $\mathfrak{X}$ is a bounded interval on $\mathbb{R}$, a functional central limit theorem holds for functions $f$ such that $|f(x)-f(y)| \leq K|x-y|^{s}$ with $s \in(1 / 2,1]$. By Adams and Fournier (2003, Theorem 1.34) the function class $C^{s}(\mathfrak{X})$ with $s \in(1 / 2,1)$ contains the Lipschitz functions with $s \in(1 / 2,1)$. Then, Corollary 10 can be used to establish a functional central limit theorem for Lipschitz functions and for stationary $\beta$-mixing processes that satisfy Condition (10). Note that when $s \in(1 / 2,1)$ and $\mathfrak{X}$ is a bounded interval, it follows that for $d=1$ the condition $s / d>1 / 2$ is satisfied.

When $\vartheta \leq 0$ such that $\lim _{\|x\|} f(x) \rightarrow 0$ does not hold, a more specific result can be given for functions in $C^{s}\left(\mathbb{R}^{d}, \vartheta\right)$ as long as one is willing to impose additional conditions on the rate of decay of $\beta_{m}$. This is done in the following corollary.

Corollary 11 Let $\chi_{t}$ be strictly stationary and $\beta$-mixing. Assume that for some $r>1$, $\sum_{m=1}^{\infty} m^{1 /(r-1)} \beta_{m}<\infty$ holds. Assume that $\vartheta \in \mathbb{R}, \vartheta \leq 0$ and $s>0$. Further assume that $\mathcal{F} \subset C^{s}\left(\mathbb{R}^{d}, \vartheta\right)$ is nonempty and bounded. Assume that for some $\gamma>0$ such that $r(\gamma-\vartheta)>1$ it holds that that $E\left[\left\|\chi_{t}\right\|^{2 r(\gamma-\vartheta)}\right]<\infty$ and that either

(i) $\gamma>s$ and $s / d>1 / 2$ or

(ii) $\gamma<s$ and $\gamma / d>1 / 2$.

Then, $v_{n}(f) \rightsquigarrow v(f)$ where $v(f)$ is a Gaussian process with covariance function $\Gamma$ and a.s. uniformly continuous sample paths.

Corollary [1] should only be applied to cases where $\vartheta \leq 0$. As for previous results, when $\vartheta>0$, the functional central limit theorem can be established under weaker assumptions.

The results in DMR are stated in general terms and form the basis for what is derived here. Nevertheless, on p.403-405 DMR provide a number of different approaches that can be used to replace high level assumptions with more primitive conditions. These methods do not lead to the sharpest possible results as far as conditions on $\beta_{m}$ are concerned for the classes of functions considered by Rio (2013). For functions whose tail decay is well controlled by a polynomial or for functions that are restricted to a bounded domain Theorem 4 also delivers sharper results. In particular, Theorem 4 shows that $\vartheta>0$, i.e. when tail 
behavior is controlled by polynomials, the functional CLT can be obtained without requiring the additional moment bound in (5). As a result, neither the marginal distribution of $\chi_{t}$ nor the dependence of the process need further restrictions beyond Condition (11). On the other hand, the results in DMR lead to similar conditions as the ones given in Theorem 6 for spaces where $\vartheta \leq 0$. The following result illustrates this. By exploiting condition (2.11) in DMR and applying Theorem 1 in Nickl and Pötscher (2007) one obtains the following.

Theorem 12 Let $1 \leq p \leq \infty, 1 \leq q \leq \infty, \vartheta \in \mathbb{R}$ and $s-d / p>0$. For $1<r<\infty$ let $\chi_{t}$ be a strictly stationary, absolutely regular process such that $\sum_{m=1}^{\infty} m^{1 /(r-1)} \beta_{m}<\infty$. Assume that for some $\gamma>0$ such that $r(\gamma-\vartheta)>1$ the moment bound

$$
\left\|\left\langle\chi_{t}\right\rangle^{(\gamma-\vartheta) / 2}\right\|_{2 r, P}<\infty
$$

holds. Let $\mathcal{F}$ be a bounded subset of $B_{p q}^{s}\left(\mathbb{R}^{d}, \vartheta\right)$. Furthermore one of the conditions holds: i) $\gamma>s-d / p$ and $1 / 2<s / d$

ii) $\gamma<s-d / p$ and $1 / 2<\gamma / d+1 / p$.

Then $v_{n}(f) \rightsquigarrow v(f)$ where $v(f)$ is a Gaussian process with covariance function $\Gamma$ and a.s. uniformly continuous sample paths.

The conditions of Theorem 12 are the same as given in Theorem 6 for the case when $\vartheta \leq 0$. However, the limitation of Theorem 12 over Theorems 4 and 6 is that it does not deliver a functional central limit theorem under the minimal condition (11) when $\vartheta>0$.

\section{Application: A Hausman Test for Linearity}

This section considers the problem of testing the specification of the conditional mean $g(x)=E[y \mid x]$ for a process $\chi_{t}=\left(y_{t}, x_{t}\right)$. The purpose of the section is to illustrate how the central limit theory developed in this paper can be used to obtain limiting results for fairly general classes of processes and conditional mean functions. Because unbounded domains are important in time series applications, the theory for weighted function spaces is particularly relevant. Minimal dependence conditions in (11) could be obtained under the additional assumption that the domain of $\chi_{t}$ is bounded. This is an immediate consequence of results in earlier sections and is only noted in passing.

The insights underlying the Hausman (1978) test are ingenious and have found applications to a large number of testing problems in econometrics. For the particular case considered in this paper the idea is to estimate the conditional mean by a linear regression 
of $y_{t}$ on $x_{t}$. The estimator is generally not consistent for the average partial derivative of the conditional mean function if the conditional expectation is non-linear. An alternative estimator uses sieve basis functions to non-parametrically estimate the possibly non-linear regression. The average derivative of this estimator is consistent even if the conditional expectation is non-linear. Thus, under the null of linearity, both estimators should converge to the same parameter. Under the alternative only the second estimator is consistent while the first estimator will be asymptotically biased under local alternatives. The Hausman test exploits these differences in asymptotic behavior by looking at the difference between the two estimators. Under the null, the test statistic has a well defined limiting distribution, while under alternatives the difference between the estimators persists, thus lending power to the test.

Comparing two competing estimators for alternative specifications of average partial derivatives is appealing from an applied perspective. The test directly answers the question of whether it is worthwhile to employ more sophisticated procedures for the estimation of average partial effects or if a simple linear regression approach is sufficient.

There is a large literature in econometrics and statistics on specification testing for the conditional mean. Tests against specific alternatives were considered for example by Cox (1961), Quandt (1974) and Davidson and McKinnon (1981). Ramsey (1969) and Newey (1985) consider tests of the orthogonality condition in a regression model while Hausman (1978) and White (1981) consider model specification tests based on the comparison of two estimators. Nonparametric tests which have power against a wider range of alternatives include Bierens (1982), Wooldridge (1992), Yatchew (1992), Zheng (1996) and Fan and Li (1996). Bierens $(1982,1987)$ points out that the tests of Hausman (1978) and White (1981) have power and in some cases consistency properties that depend on the choice of the estimator that is consistent under both the null and the alternative. The test considered in this section is pointwise consistent against all fixed non-parametric deviations $h$ in the class $B_{\infty \infty}^{s}\left(\mathbb{R}^{d}, \vartheta\right)$ with $\operatorname{Cov}(h, x)+\pi(h) \neq 0$. The parameter $\pi(h)$ captures the discrepancy between average partial effects when the model is linear and when it is non-linear. The term $\operatorname{Cov}(h, x)$ accounts for linear regression bias under the alternative. Under the null of a linear conditional mean the local deviation $h$ is zero and $\pi(h)=0$.

The test proposed in this study has non-trivial power against local alternatives of the form $n^{-1 / 2} h(x)$ for fixed $h(x) \in B_{\infty \infty}^{s}\left(\mathbb{R}^{d}, \vartheta\right)$ with $\operatorname{Cov}(h, x)+\pi(h) \neq 0$. Horowitz and Spokoiny (2001) point out that the tests of Bierens (1982), Andrews (1997) and Bierens and Ploberger (1997) have non-trivial power against such alternatives while the tests of 
Wooldridge (1992), Yatchew (1992), Zheng (1996) and Fan and Li (1996) only have nontrivial power against alternatives that are local at rates slower than $n^{-1 / 2}$. Horowitz and Spokoiny (2001) develop tests that have power against more general alternatives $n^{-1 / 2} h_{n}(x)$ where $h_{n}(x)$ is a sequence of functions. Their tests have power uniformly against certain smooth alternatives against which the test in this paper and the tests of Bierens (1982), Andrews (1997) and Bierens and Ploberger (1997) do not have non-trivial power. Nevertheless, the appeal of the test proposed in this paper is its simplicity in terms of implementation and interpretation.

The estimation problem considered in this study is semi-parametric in nature. The distribution of the test statistic depends on the non-parametric functional estimated by the second estimator. The influence function of the test statistic defines an empirical process that can be used to obtain the limiting distribution under the null and under local alternatives. This is now formalized.

Let $\chi_{t}=\left(y_{t}, x_{t}\right) \in \mathbb{R}^{2}$ be a strictly stationary $\beta$-mixing process and define $g\left(x_{t}\right)=$ $E\left[y_{t} \mid x_{t}\right]$. Extensions to multivariate $x_{t}$ are straight forward but omitted for ease of exposition. Consider testing the hypothesis that $g(x)=\psi_{0}+\psi_{1} x$ against the alternative that $g(x)$ is a non-linear function of $x$. A linear regression estimator for $\psi_{1}$ is generally inconsistent if $g(x) \neq \psi_{0}+\psi_{1} x$. A Hausman test is then based on the squared difference for two estimators of $E\left[\partial g\left(x_{t}\right) / \partial x\right]$. Under the null, the average partial effect is simply $\psi_{1}$ which is estimated as a regression of $y_{t}$ on a constant and $x_{t}$. Under the alternative, $E\left[\partial g\left(x_{t}\right) / \partial x\right]$ is estimated by a plug-in series estimator for $g(x)$.

Define $P^{\kappa}(z)=\left(p_{1 \kappa}(z), \ldots, p_{\kappa \kappa}(z)\right)^{\prime}$, where $p_{1 \kappa}(z)=z$ for all $\kappa, \mu_{P}^{\kappa}=E\left[P^{\kappa}\left(z_{t}\right)\right]$ and $\tilde{P}^{\kappa}(z)=P^{\kappa}(z)-\mu_{P}^{\kappa}$. Define $P=\left[P^{\kappa}\left(x_{1}\right), \ldots, P^{\kappa}\left(x_{n}\right)\right]^{\prime}$,

$$
M P=\left[P^{\kappa}\left(x_{1}\right)-\bar{P}^{\kappa}, \ldots, P^{\kappa}\left(x_{n}\right)-\bar{P}^{\kappa}\right]^{\prime}
$$

where $M=I_{n}-n^{-1} \mathbf{1}_{n} \mathbf{1}_{n}^{\prime}$ with $\mathbf{1}_{n}$ a vector of length one composed of the element one and $\bar{P}^{\kappa}=n^{-1} \sum_{t=1}^{n} P^{\kappa}\left(x_{t}\right)$. The series estimator for $E[y \mid x]$ is $\hat{g}_{\kappa}(x)=\hat{\psi}_{0, \kappa}+P^{\kappa}(x) \hat{\psi}_{\kappa}$ where $\hat{\psi}_{\kappa}=\left(P^{\prime} M P\right)^{-1} P^{\prime} M y$. The estimator for the constant is given by $\hat{\psi}_{0, \kappa}=\bar{y}-\bar{P}^{\kappa} \hat{\psi}_{\kappa}$ with $\bar{y}=n^{-1} \sum_{t=1}^{n} y_{t}$.

Let $\theta=\left(\theta_{l}, \theta_{n l}\right)$ where $\theta_{l}$ is the average partial effect under the linear specification and $\theta_{n l}=E\left[\partial g\left(x_{t}\right) / \partial x\right]$ is the average partial effect under the non-linear specification. An estimator for $\theta$ is based on a Z-estimator 1 using a plug in non-parametric estimate

\footnotetext{
${ }^{1}$ This terminology appreas for example in van der Vaart (1998, p 41).
} 
$\hat{g}_{k}=\hat{g}_{k}(x)$. For this purpose define the moment function

$$
\hat{m}\left(\chi_{t}, \theta, \hat{g}_{\kappa}\right)=\left[\begin{array}{c}
\left(y_{t}-\bar{y}-\theta_{l}\left(x_{t}-\bar{x}\right)\right)\left(x_{t}-\bar{x}\right) \\
\frac{\partial P^{\kappa}\left(x_{t}\right)^{\prime}}{\partial x} \hat{\psi}_{\kappa}-\theta_{n l}
\end{array}\right]
$$

and let

$$
m_{n}(\theta)=n^{-1} \sum_{t=1}^{n} \hat{m}\left(\chi_{t}, \theta, \hat{g}_{\kappa}\right) .
$$

The Z-estimator $\hat{\theta}_{\kappa}=\left(\hat{\theta}_{l}, \hat{\theta}_{n l}\right)$ is obtained by solving $m_{n}\left(\hat{\theta}_{\kappa}\right)=0$. A Hausman test of linearity then compares the two estimators by forming the test statistic

$$
\left(\hat{\theta}_{l}-\hat{\theta}_{n l}\right)^{2} / \widehat{\operatorname{Var}}\left(\hat{\theta}_{l}-\hat{\theta}_{n l}\right) .
$$

The estimator $\hat{\theta}_{l}$ is not usually efficient under the null. However, it is well known that the Hausman testing principle can still be applied, albeit at the cost of requiring more complicated expressions for $\operatorname{Var}\left(\hat{\theta}_{l}-\hat{\theta}_{n l}\right)$. The limiting distribution of $\hat{\theta}_{l}-\hat{\theta}_{n l}$ can be analyzed within the framework of Newey (1994). The results of Newey (1994) show that non-parametric estimation of $g(x)$ does affect the limiting distribution of $\hat{\theta}_{l}-\hat{\theta}_{n l}$, but in ways that do not depend on the specific form of the estimator for $g(x)$.

The limiting distribution of the test statistic is analyzed for the following data-generating mechanism under local alternatives $g_{h}(x)$,

$$
y_{t}=\psi_{0}+\psi_{1} x_{t}+\frac{h\left(x_{t}\right)}{\sqrt{n}}+u_{t}
$$

where $g_{h}(x)=\psi_{0}+\psi_{1} x_{t}+n^{-1 / 2} h\left(x_{t}\right)$ and $u_{t}=y_{t}-E\left[y_{t} \mid x_{t}\right]$ is such that $E\left[u_{t} \mid x_{t}\right]=0$. Assume that $h(x)=h \in B_{\infty \infty}^{s+1}(\mathbb{R}, \vartheta)$ for some $s>1 / 2$ and some $\vartheta \in \mathbb{R}$. Let $E\left[x_{t}\right]=\mu_{x}$ and set

$$
\tilde{b}(h)=[b(h), 0]^{\prime}
$$

with $b(h)=E\left[\left(x_{t}-\mu_{x}\right) h\left(x_{t}\right)\right]$. The term $b(h)$ captures biases in estimating $\psi_{1}$ with a linear regression when $h \neq 0$. Under the null of a linear conditional mean the function $h$ is $h_{0}\left(x_{t}\right)=0$ which implies that $b\left(h_{0}\right)=0$. Let

$$
Q=E\left[\partial m\left(\chi_{t}, \theta, g_{h}\right) / \partial \theta\right]=\left[\begin{array}{cc}
\sigma_{x}^{2} & 0 \\
0 & 1
\end{array}\right]
$$

where $\sigma_{x}^{2}=E\left[\left(x_{t}-\mu_{x}\right)^{2}\right]$. Let $m\left(\chi_{t}, \theta, g_{h}\right)$ be the population analog of $\hat{m}\left(\chi_{t}, \theta, g_{h}\right)$ defined in (7) where in $m($.$) the empirical estimates \bar{x}$ and $\bar{y}$ are replaced with $\mu_{x}$ and $\mu_{y}$. Let $\theta_{0}=\left(\psi_{1}, \theta_{n l}\right)^{\prime}$ be the value of $\theta$ for the true data generating process (9) under local 
alternatives. Under regularity conditions it follows from arguments similar to Newey (1994) that for $h$ fixed,

$$
\sqrt{n}\left(\hat{\theta}_{\kappa}-\theta_{0}\right)=Q^{-1}\left(n^{-1 / 2} \sum_{t=1}^{n}\left(m\left(\chi_{t}, \theta_{0}, g_{h}\right)+\gamma\left(\chi_{t}\right)\right)\right)+o_{p}(1) .
$$

The correction term $\gamma\left(\chi_{t}\right)$ accounts for non-parametric estimation of the nuisance parameter $g_{h}$ and can be derived using the methods developed in Newey (1994). It is given by

$$
\gamma\left(\chi_{t}\right)=\left[\begin{array}{c}
0 \\
\delta_{n l}\left(x_{t}\right)
\end{array}\right]\left(y_{t}-\psi_{0}-\psi_{1} x_{t}-\frac{h\left(x_{t}\right)}{\sqrt{n}}\right)
$$

where $\delta_{n l}\left(x_{t}\right)=-\zeta_{x}(x)^{-1} \partial \zeta_{x}(x) / \partial x$ and $\zeta_{x}(x)$ is the marginal density of $x_{t}$, see Newey (1994, p.1362) or Hardle and Stoker (1989). Define the empirical process

$$
v_{n}(h)=n^{-1 / 2} \sum_{t=1}^{n}\left(m\left(\chi_{t}, \theta_{0}, g_{h}\right)+\gamma\left(\chi_{t}\right)-E\left[m\left(\chi_{t}, \theta_{0}, g_{h}\right)\right]\right)
$$

The central limit theorems developed in the first part of the paper play a dual role in analyzing the limiting properties of $\hat{\theta}_{\kappa}$. On the one hand, stochastic equicontinuity properties of the empirical process (11) can be used to verify regularity conditions in Newey (1994). On the other hand, the functional central limit theorem delivers a stochastic process representation of the limiting distribution of $\hat{\theta}_{\kappa}$ over the class of local alternatives.

Condition 1 Let $\chi_{t}$ be a strictly stationary and $\beta$-mixing process. Assume that (1) holds. Assume that for some $\vartheta \in \mathbb{R}, \mathcal{F} \subset B_{\infty \infty}^{s+1}\left(\mathbb{R}^{d}, \vartheta\right)$ is nonempty and bounded, $0 \in \mathcal{F}$ and $h \in \mathcal{F}$. Let $\zeta_{x}(x)$ be the marginal density of $x_{t} . \zeta_{x}(x)$ is absolutely continuous with respect to Lebesgue measure, is continuously differentiable with derivative $\partial \zeta_{x}(x) / \partial x$ vanishing as $x \rightarrow \pm \infty$ and $\zeta_{x}(x)^{-1} \partial \zeta_{x}(x) / \partial x \in \mathcal{F}$. Assume that one of the following conditions hold: (i) $\vartheta \leq-1$ and for some $\gamma>0$ it follows that $\left\|\left\langle\chi_{t}\right\rangle^{(\gamma-\vartheta-1) / 2}\right\|_{2, \beta}<\infty, \gamma>s$ and $s>1 / 2$; (ii) $\vartheta \leq-1$ and for some $\gamma>0$ it follows that $\left\|\left\langle\chi_{t}\right\rangle^{(\gamma-\vartheta-1) / 2}\right\|_{2, \beta}<\infty, \gamma<s$ and $\gamma>1 / 2$.

Condition 11 directly leads to the following lemma, which is an immediate consequence of Theorem 4, Let

$$
v_{t}=\left[\begin{array}{c}
u_{t}\left(x_{t}-\mu_{x}\right) \\
\frac{\partial g_{h}\left(x_{t}\right)}{\partial x}-\theta_{n l}+\delta_{n l}\left(x_{t}\right) u_{t}
\end{array}\right]
$$

and $\Gamma(h)=\sum_{j=-\infty}^{\infty} E\left[v_{t} v_{t-j}^{\prime}\right]$. 
Lemma 13 Assume that Condition 1 and 0 hold. Let $v_{n}(h)$ be defined in (11). Then, $v_{n}(h) \rightsquigarrow v(h)$ where $v(h)$ is a Gaussian process with covariance function $\Gamma(h)$ and a.s. uniformly continuous sample paths.

The following high level regularity conditions are similar to conditions imposed in Newey (1994). Since this section is mostly meant to highlight the usefulness of the functional central limit theory discussed in this paper the regularity conditions are high level with regard to the semiparametric estimators. A full development of these estimators is beyond the scope of this paper.

Condition 2 Let $u_{t}=y_{t}-E\left[y_{t} \mid x_{t}\right]$. Then, i) $E\left[u_{t}^{2} \mid x_{t}\right]=\sigma_{t}^{2}\left(x_{t}\right)$ and

$$
E\left[\sigma_{t}^{2}\left(x_{t}\right) \zeta_{x}(x)^{-2}\left(\partial \zeta_{x}(x) / \partial x\right)^{2}\right]<\infty .
$$

ii) Let $\hat{g}$ be a series estimator of $g_{h}$. Then, there exists a sequence $\kappa=\kappa_{n}$ such that $\kappa_{n} \rightarrow \infty$ as $n \rightarrow \infty$ and $\sqrt{n}\left\|\hat{g}-g_{h}\right\|_{2, \beta}^{2}=o_{p}(1)$.

iii) $1 / \sqrt{n} \sum_{t=1}^{n} \partial\left(\hat{g}\left(x_{t}\right)-g_{h}\left(x_{t}\right)\right) / \partial x-\gamma\left(x_{t}\right)=o_{p}(1)$.

The next lemma establishes the limiting process for the empirical moment function $m_{n}\left(\theta_{\kappa}\right)$.

Lemma 14 Assume that Conditions 1 and Q hold. Let $m_{n}\left(\theta_{0}\right)$ be defined in (8). Then, for $h \in \mathcal{F}$ fixed,

$$
\sqrt{n} m_{n}\left(\theta_{0}\right)=v_{n}(h)+\tilde{b}(h)+o_{p}(1)
$$

and

$$
\sqrt{n} m_{n}\left(\theta_{0}\right) \rightarrow_{d} v(h)+\tilde{b}(h)
$$

where $v(h)$ is a Gaussian process with covariance function $\Gamma(h)$ and a.s. uniformly continuous sample paths. The bias term $\tilde{b}(h)$ is defined in (10).

The following condition is needed to derive an asymptotic limiting distribution of the estimators for $\theta_{l}$ and $\theta_{n l}$. The estimators exist in closed form which greatly simplifies their analysis. For the representation of the limiting distribution it is useful to partition $P=$ $\left[P_{1}, P_{2}\right]$ where $P_{1}=\left[x_{1}, \ldots, x_{n}\right]^{\prime}$ and $P_{2}=\left[\left(p_{2 \kappa}\left(x_{1}\right), \ldots, p_{\kappa \kappa}\left(x_{1}\right)\right)^{\prime}, \ldots,\left(p_{2 \kappa}\left(x_{n}\right), \ldots, p_{\kappa \kappa}\left(x_{n}\right)\right)^{\prime}\right]^{\prime}$. Then, following Newey (1994, p.1374) an explicit formula for $\hat{\theta}_{\kappa}$ is given as

$$
\hat{\theta}_{\kappa}=\hat{Q}^{-1}\left[\begin{array}{c}
P_{1}^{\prime} M y \\
\hat{\Psi}^{\prime}\left(P^{\prime} P\right)^{-1} P^{\prime} y
\end{array}\right]
$$


where $\hat{\Psi}=n^{-1} \sum_{t=1}^{n} \partial P^{\kappa}\left(x_{t}\right) / \partial x$ and

$$
\hat{Q}=\left[\begin{array}{cc}
n^{-1} \sum_{t=1}^{n}\left(x_{t}-\bar{x}\right)^{2} & 0 \\
0 & 1
\end{array}\right] .
$$

The following additional high level conditions are imposed.

Condition 3 i) For $\kappa$ as specified in Condition 0 it follows that

$$
\hat{Q}=n^{-1} \sum_{t=1}^{n}\left[\begin{array}{cc}
\left(x_{t}-\bar{x}\right)^{2} & 0 \\
0 & 1
\end{array}\right] \rightarrow_{p} Q=\left[\begin{array}{cc}
\sigma_{x}^{2} & 0 \\
0 & 1
\end{array}\right]
$$

where $\sigma_{x}^{2}=\operatorname{Var}\left(x_{t}\right)$ and $Q$ is a fixed, positive definite matrix that does not depend on $g$. ii) It follows that

$$
\sup _{g \in \mathcal{F}}\left\|n^{-1 / 2} \sum_{t=1}^{n} \hat{m}\left(\chi_{t}, \theta_{0}, g\right)-m\left(\chi_{t}, \theta_{0}, g\right)\right\|=o_{p}(1) .
$$

iii) Assume that $n^{-1} \sum_{t=1}^{n}\left(x_{t}-\bar{x}\right) h\left(x_{t}\right)=b(h)+o_{p}(1)$.

The asymptotic limiting distribution of the estimators for $\theta_{l}$ and $\theta_{n l}$ under the null of $h=0$ and local alternatives is stated in the next lemma. This distribution then is used to determine critical values for the Hausman test statistic.

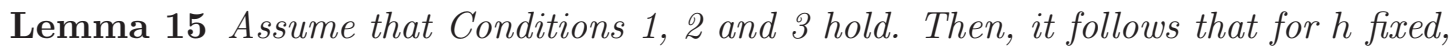

$$
\sqrt{n}\left(\hat{\theta}_{\kappa}-\theta_{0}\right) \rightarrow_{d} Q^{-1}(v(h)+\tilde{b}(h))
$$

where $\tilde{b}\left(h_{0}\right)=0$ and $Q^{-1} v(h) \sim N\left(0, Q^{-1} \Gamma(h) Q^{-1}\right)$. If in addition, $E\left[u_{t} \mid \mathcal{A}^{t-1}\right]=0$ and $E\left[u_{t}^{2} \mid x_{t}\right]=\sigma^{2}$ where $\sigma^{2}$ is constant and $\sigma^{2}>0$, then it follows that $Q^{-1} v(h) \sim$ $N\left(0, \sigma^{2} Q^{-1} \Lambda(h) Q^{-1}\right)$ where $\Lambda(h)$ is defined as $\Lambda(h)=E\left[v_{t} v_{t}^{\prime}\right]$.

To form the Hausman statistic assume that $\hat{\Gamma}$ is a consistent estimator of $\Gamma$ and $\hat{Q}$ is consistent for $Q$ by Condition 3 , Let $e=(1,-1)^{\prime}$. A generalized Hausman statistic to test the null hypothesis of a linear conditional mean then is given as

$$
\hat{H}_{1}=\frac{n\left(\hat{\theta}_{l}-\hat{\theta}_{n l}\right)^{2}}{e^{\prime} \hat{Q}^{-1} \hat{\Gamma} \hat{Q}^{-1} e}
$$

If the additional conditions imposed on $u_{t}$ in Lemma 15 hold then the test statistic can be simplified to

$$
\hat{H}_{2}=\frac{n\left(\hat{\theta}_{l}-\hat{\theta}_{n l}\right)^{2}}{\left(e^{\prime} \hat{Q}^{-1} \hat{\Lambda} \hat{Q}^{-1} e\right)} .
$$


The limiting distributions of the two Hausman statistics are summarized in the following Theorem.

Theorem 16 Assume that Conditions 1, 回and可hold. Let $\pi(h)=\psi_{1}-\theta_{n l}=-E\left[\partial h\left(x_{t}\right) / \partial x\right]$. Then, $\hat{H}_{1}$ defined in (12) converges (pointwise for $h$ fixed) to a non-central $\chi^{2}$ process

$$
\hat{H}_{1} \rightarrow_{d} \chi_{1}^{2}\left(\tilde{\lambda}_{1}\right)
$$

where for fixed $h, \chi_{1}^{2}\left(\tilde{\lambda}_{1}\right)$ is a non-central chi-square distribution with one degree of freedom and non-centrality parameter $\tilde{\lambda}_{1}$ and

$$
\tilde{\lambda}_{1}=\frac{b(h)+\pi(h)}{\sigma_{x}^{2} \sqrt{e^{\prime} Q^{-1} \Gamma(h) Q^{-1} e}} .
$$

If in addition, $E\left[u_{t} \mid \mathcal{A}^{t-1}\right]=0$ and $E\left[u_{t}^{2} \mid x_{t}\right]=\sigma^{2}$ where $\sigma^{2}$ is constant and $\sigma^{2}>0$, then it follows that

$$
\hat{H}_{1} \rightarrow_{d} \chi_{1}^{2}\left(\tilde{\lambda}_{2}\right), \hat{H}_{2} \rightarrow_{d} \chi_{2}^{2}\left(\tilde{\lambda}_{2}\right)
$$

where the non-centrality parameter $\tilde{\lambda}_{2}$ is given by

$$
\tilde{\lambda}_{2}=\frac{b(h)+\pi(h)}{\sigma_{x}^{2} \sqrt{e^{\prime} Q^{-1} \Lambda(h) Q^{-1} e}} .
$$

Theorem 16 establishes that under the null hypothesis of a linear conditional mean of $y_{t}$ the limiting distribution of $\hat{H}_{1}$ and, under additional conditions, of $\hat{H}_{2}$ are asymptotically $\chi_{1}^{2}$. For a significance level $\alpha$, let $c_{\alpha}$ be the critical value of the central $\chi_{1}^{2}$ distribution, i.e. $\alpha=\operatorname{Pr}\left(\chi_{1}^{2}>c_{\alpha}\right)$. The null hypothesis of a linear conditional mean then is rejected if $\hat{H}_{1}>c_{\alpha}$ or $\hat{H}_{2}>c_{\alpha}$.

The analysis in Theorem 16 also shows how the power of the test against local alternatives depends on the local alternative $h$ and the marginal distribution of $x_{t}$. The term $b(h)$ captures the bias in estimating the coefficient $\psi_{1}$ of the linear term in $g(x)$ by linear regression. The term $\pi(h)$ captures the discrepancy between the two estimators due to the difference between $\psi_{1}$ and $\theta_{n l}$. The asymptotic power function of the test is given by $\operatorname{Pr}\left(\chi_{1}^{2}\left(\tilde{\lambda}_{1}\right)>c_{\alpha}\right)$ as $h$ ranges over the set of permissible alternatives.

\section{Conclusion}

The paper combines recent results on bracketing numbers for weighted Besov spaces with a functional central limit theorem for strictly stationary $\beta$-mixing processes. It is shown that by specializing the bracketing results to a particular Hilbert space of relevance to the 
dependent limit theory, functional central limit theorems for dependent processes indexed by Besov classes can be obtained directly. These insights lead to some new results in function spaces with polynomially decaying functions over unbounded domains and smooth functions over bounded domains.

It is shown how the limit theory can be used to simplify some proofs in the analysis of semiparametric estimators and tests. An example of a Hausman test for linearity is considered in detail. More specifically, the central limit theorem implies a stochastic equicontinuity property that helps shorten arguments needed to establish the limiting behavior of the test. The central limit theory also allows to represent the limiting distribution over a class of local alternatives under general conditions. Finally, a comparison of two versions of the test when stronger conditions on the model are imposed is provided.

A number of the conditions imposed in Section 5 are high level. A detailed analysis of non-parametric estimation in weighted Besov spaces is beyond the scope of the paper and left for future research. 


\section{A Proofs}

Proof of Theorem 2. The proof follows the argument in Nickl and Pötscher (2007, p.184). Let $N\left(\delta, \mathcal{F},\|\cdot\|_{\infty}\right)$ be the minimal covering number of $\mathcal{F}$ with respect to $\|\cdot\|_{\infty}$ and $H\left(\delta, \mathcal{F},\|\cdot\|_{\infty}\right)=\log N\left(\delta, \mathcal{F},\|\cdot\|_{\infty}\right)$ the metric entropy for $\mathcal{F}$. From Nickl and Pötscher (2007, p.184, Eq.3) it follows that for all $\vartheta \in \mathbb{R}$ and all $\gamma>0$

$$
H\left(\delta, \mathcal{F},\left\|\langle x\rangle^{(\vartheta-\gamma) / 2}\right\|_{\infty}\right) \precsim\left\{\begin{array}{cc}
\delta^{-d / s} & \text { if } \gamma>s-d / p \\
\delta^{-(\gamma / d+1 / p)^{-1}} & \text { if } \gamma<s-d / p
\end{array}\right.
$$

Let $B_{i}$ be closed balls in $C\left(\mathbb{R}^{d},\langle x\rangle^{(\vartheta-\gamma) / 2}\right)=\left\{f: f(.)\langle x\rangle^{(\vartheta-\gamma) / 2} \in C\left(\mathbb{R}^{d}\right)\right\}$ with radius $\delta$ (relative to the norm $\left\|(.)\langle x\rangle^{(\vartheta-\gamma) / 2}\right\|_{\infty}$ ) covering $\mathcal{F}$. Note that the number of such balls is $N\left(\delta, \mathcal{F},\left\|(.)\langle x\rangle^{(\vartheta-\gamma) / 2}\right\|_{\infty}\right)$. Let $f_{i}$ be the center of $B_{i}$. Then each $B_{i}$ contains the functions $f$ such that

$$
\sup _{x \in \mathbb{R}^{d}}\left|f(x)-f_{i}(x)\right|\langle x\rangle^{(\vartheta-\gamma) / 2} \leq \delta .
$$

The brackets

$$
\left[f_{i}(x)-\delta\langle x\rangle^{(\gamma-\vartheta) / 2}, f_{i}(x)+\delta\langle x\rangle^{(\gamma-\vartheta) / 2}\right]
$$

are contained in $B_{i}$ and cover $\mathcal{F}$. The $\mathcal{L}_{2, \beta}(P)$ norm of these brackets is

$$
\left\|2 \delta\langle x\rangle^{(\gamma-\vartheta) / 2}\right\|_{2, \beta} .
$$

First consider the case when $\vartheta>0$. In that case one can choose $\gamma=\vartheta$. Then, $\left\|2 \delta\langle x\rangle^{(\gamma-\vartheta) / 2}\right\|_{2, \beta}=$ $\|2 \delta\|_{2, \beta}$. Now note that for the constant function $\delta$

$$
Q_{\delta}(u)=\inf (t: P(|\delta|>t) \leq u)=\delta
$$

such that

$$
\|2 \delta\|_{2, \beta}^{2}=\sum_{m=0}^{\infty} \int_{0}^{\beta_{m}}\left(Q_{2 \delta}(u)\right)^{2} d u=(2 \delta)^{2} \sum_{m=0}^{\infty} \beta_{m}<\infty
$$

by Condition (11). One obtains from Nickl and Pötscher (2007, p.184, eq. 4) that

$$
H_{[]}\left(2 \delta \sum_{m=0}^{\infty} \beta_{m}, \mathcal{F},\|\|_{2, \beta}\right) \leq H\left(\delta, \mathcal{F},\|\|_{\infty}\right)
$$

such that the result follows immediately from (14).

When $\vartheta \leq 0$ the brackets have size

$$
2 \delta\left\|\langle x\rangle^{(\gamma-\vartheta) / 2}\right\|_{2, \beta}<\infty
$$


which is bounded by the conditions of the Theorem. It follows again by Nickl and Pötscher (2007, p.184, eq. 4) that

$$
H_{[]}\left(2 \delta\left\|\langle x\rangle^{(\gamma-\vartheta) / 2}\right\|_{2, \beta}, \mathcal{F},\|\|_{2, \beta}\right) \leq H\left(\delta, \mathcal{F},\left\|(.)\langle x\rangle^{(\vartheta-\gamma) / 2}\right\|_{\infty}\right) .
$$

Then, (14) delivers the stated result.

Proof of Corollary 3. From the proof of Theorem 2 the $\mathcal{L}_{2, \beta}(P)$ norm of the brackets is, for all $\gamma>0$ and all $\vartheta \in \mathbb{R}$,

$$
\left\|2 \delta\langle x\rangle^{(\gamma-\vartheta) / 2}\right\|_{2, \beta} \leq 2 \delta M^{(\gamma-\vartheta) / 2} \sum_{m=0}^{\infty} \beta_{m}<\infty .
$$

Therefore, the bound in (15) can be applied and the result again follows by (14).

Proof of Theorem 4. The result follows from Theorem 1 in DMR once all of their conditions are verified. First show that $\mathcal{F} \in \mathcal{L}_{2, \beta}(P)$. Let $\mathcal{L}(\beta)$ be the class of integer valued random variables with distribution function $G_{\beta}(n)=1-\beta_{n}$ for any $n \in \mathbb{N}$ (see DMR, p. 423). For any $b \in \mathcal{L}(\beta)$ and some real number $K>0$ it follows that

$$
\begin{aligned}
E\left[b f^{2}\left(\chi_{t}\right)\right] & =E\left[b\left\langle\chi_{t}\right\rangle^{-\vartheta}\left(f\left(\chi_{t}\right)\left\langle\chi_{t}\right\rangle^{\vartheta / 2}\right)^{2}\right] \\
& \leq\left(\sup _{x \in \mathbb{R}^{d} f \in \mathcal{F}}\left|f(x)\langle x\rangle^{\vartheta / 2}\right|\right)^{2} E\left[b\left\langle\chi_{t}\right\rangle^{-\vartheta}\right] \\
& \leq K^{2} E\left[b\left\langle\chi_{t}\right\rangle^{-\vartheta}\right]
\end{aligned}
$$

where the first inequality is obtained by applying Proposition 3 of Nickl and Pötscher (2007) and because $f(x)\langle x\rangle^{\vartheta / 2} \in \mathcal{F}$ by assumption. For any $f \in \mathcal{F}$ it follows from DMR, Eq. (6.2) and

$$
\begin{aligned}
\|f\|_{2, \beta} & =\sup _{b \in \mathcal{L}(\beta)} \sqrt{E\left[b f^{2}\left(\chi_{t}\right)\right]} \\
& \leq K \sup _{b \in \mathcal{L}(\beta)} \sqrt{E\left[b\left\langle\chi_{t}\right\rangle^{-\vartheta}\right]}
\end{aligned}
$$

where the inequality uses (16). If $\vartheta \geq 0$ the inequality

$$
\left\langle\chi_{t}\right\rangle^{-\vartheta} \leq 1
$$

together with $b \geq 0$ leads to

$$
\|f\|_{2, \beta} \leq K \sup _{b \in \mathcal{L}(\beta)} \sqrt{E[b]}=K\|1\|_{2, \beta}=K \sqrt{\sum_{m=0}^{\infty} \beta_{m}} .
$$


When $\vartheta<0$, (17) leads to

$$
\|f\|_{2, \beta} \leq K\left\|\left\langle\chi_{t}\right\rangle^{-\vartheta}\right\|_{2, \beta}
$$

Since in this case,

$$
\left\langle\chi_{t}\right\rangle^{-\vartheta} \geq 1
$$

and for any $\gamma>0$,

$$
\left\langle\chi_{t}\right\rangle^{\gamma-\vartheta} \geq\left\langle\chi_{t}\right\rangle^{-\vartheta}
$$

it follows from (19) that

$$
\|f\|_{2, \beta} \leq K\left\|\left\langle\chi_{t}\right\rangle^{\gamma-\vartheta}\right\|_{2, \beta}<\infty
$$

which is bounded by assumption. Thus, (18) and (20) show that $f \in \mathcal{F} \subset B_{p q}^{s}\left(\mathbb{R}^{d}, \vartheta\right)$ with either $\vartheta \geq 0$ or $\vartheta<0$ and some $\gamma>0$ such that $\left\|\langle x\rangle^{(\gamma-\vartheta) / 2}\right\|_{2, \beta}<\infty$ implies that $\mathcal{F} \in \mathcal{L}_{2, \beta}(P)$.

It remains to be show that

$$
\int_{0}^{1} \sqrt{H_{[]}\left(\delta, \mathcal{F},\|\|_{2, \beta}\right)} d \delta<+\infty .
$$

For case (i) Theorem 2 implies that $H_{[]}\left(\delta, \mathcal{F},\|\|_{2, \beta}\right) \precsim \delta^{-d / s}$ such that (21) holds for $d / 2 s<1$. For case (ii) Theorem 2 implies that $H_{[]}\left(\delta, \mathcal{F},\|\|_{2, \beta}\right) \precsim \delta^{-(\gamma / d+1 / p)^{-1}}$ such that (21) holds for $1 / 2(\gamma / d+1 / p)^{-1}<1$. Cases (iii) and (iv) follow in the same way. This establishes the result.

Proof of Corollary [5. For any $s>d / p$ fix $\vartheta$ such that $\vartheta>s-d / p$. By construction $0<\vartheta<\infty$ and thus $f(.)\langle x\rangle^{\vartheta}$ is bounded for $x \in \mathfrak{X}$ and $f(.)\langle x\rangle^{\vartheta} \in B_{p q}^{s}(\mathfrak{X}, \vartheta)$. As in Nickl and Pötscher (2007, p.186), conclude that $\mathcal{F} \subseteq B_{p q}^{s}(\mathfrak{X}, \vartheta)$. The results of Theorem 4 can now be applied. In particular, using the bound in (16) leads to

$$
\|f\|_{2, \beta} \leq K \sup _{b \in \mathcal{L}(\beta)} \sqrt{E\left[b\left\langle\chi_{t}\right\rangle^{-\vartheta}\right]} \leq K M^{-\vartheta / 2} \sqrt{\sum_{m=0}^{\infty} \beta_{m}}<\infty .
$$

The result now follows from the fact that 21 holds by the results in Corollary 3 ,

Proof of Theorem 6. From DMR Lemma 2, (S.1) and p. 404 it follows for $\phi(x)=x^{r}$ with $r>1$ that

$$
\sum_{m=1}^{\infty} m^{1 /(r-1)} \beta_{m}<\infty
$$

and

$$
\left\|\left\langle\chi_{t}\right\rangle^{(\gamma-\vartheta) / 2}\right\|_{2 r, P}<\infty
$$


is sufficient for $\left\|\left\langle\chi_{t}\right\rangle^{(\gamma-\vartheta) / 2}\right\|_{2, \beta}<\infty$. Note that (23) holds since $r(\gamma-\vartheta)>1$ and by Jensen's inequality

$$
\left\|\left\langle\chi_{t}\right\rangle^{(\gamma-\vartheta) / 2}\right\|_{2 r, P}^{2 r}=E\left[\left\langle\chi_{t}\right\rangle^{r(\gamma-\vartheta)}\right] \leq 1+E\left[\left\|\chi_{t}\right\|^{2 r(\gamma-\vartheta)}\right]<\infty
$$

where the expectation on the RHS is bounded by assumption. The result now follows from Theorem 4 .

Proof of Theorem 12, The result follows from DMR (eq 2.11) and (eq. S.1). In particular, the condition

$$
\int_{0}^{1} \sqrt{H_{[]}\left(t,,\|\cdot\|_{2 p}\right) d t}<\infty
$$

needs to hold. From Nickl and Pötscher (2007) it follows that under the stated conditions in (i),

$$
H_{[]}\left(t,,\|\cdot\|_{2 p}\right) \precsim t^{-d / s}
$$

such that (24) holds as long as $d /(2 s)<1$ or $1 / 2<s / d$. Under conditions (ii) one obtains similarly that

$$
H_{[]}\left(t,,\|\cdot\|_{2 p}\right) \precsim t^{-(\gamma / d+1 / p)^{-1}}
$$

such that (24) holds as long as $r p /(\gamma p+d)<1$ or $1 / 2<(\gamma / d+1 / p)$.

Proof of Lemma 13. Recall that

$$
m\left(\chi_{t}, \theta_{0}, \hat{g}_{\kappa}\right)=\left[\begin{array}{c}
\left(y_{t}-\mu_{y}-\psi_{1}\left(x_{t}-\mu_{x}\right)\right)\left(x_{t}-\mu_{x}\right) \\
\frac{\partial P^{\kappa}\left(x_{t}\right)^{\prime}}{\partial x} \hat{\psi}_{\kappa}-\theta_{n l}
\end{array}\right]
$$

and that

$$
E\left[y_{t}\right]=\psi_{0}+\psi_{1} E\left[x_{t}\right]+\mu_{h} .
$$

where $\mu_{h}=E\left[h\left(x_{t}\right)\right]$. It follows that

$$
\begin{aligned}
v_{n}(h) & =n^{-1 / 2} \sum_{t=1}^{n}\left(m\left(\chi_{t}, \theta_{0}, g_{h}\right)+\gamma\left(\chi_{t}\right)-E\left[m\left(\chi_{t}, \theta_{0}, g_{h}\right)\right]\right) \\
& =n^{-1 / 2} \sum_{t=1}^{n}\left[\begin{array}{c}
\left(u_{t}+n^{-1 / 2}\left(h\left(x_{t}\right)-\mu_{h}\right)\right)\left(x_{t}-\mu_{x}\right) \\
\partial g_{h}\left(x_{t}\right) / \partial x-\theta_{n l}-f_{x}\left(x_{t}\right)^{-1} \partial f_{x}\left(x_{t}\right) / \partial x u_{t}
\end{array}\right] \\
& -n^{-1 / 2} \sum_{t=1}^{n}\left[\begin{array}{c}
n^{-1 / 2} E\left[\left(x_{t}-\mu_{x}\right)\left(h\left(x_{t}\right)-\mu_{h}\right)\right] \\
0
\end{array}\right] \\
& =n^{-1 / 2} \sum_{t=1}^{n}\left[\begin{array}{c}
\partial g_{h}\left(x_{t}\right) / \partial x-\theta_{n l}-\zeta_{x}\left(x_{t}\right)^{-1} \partial \zeta_{x}\left(x_{t}\right) / \partial x u_{t}
\end{array}\right] \\
& -n^{-1} \sum_{t=1}^{n}\left[\begin{array}{c}
\left(x_{t}-\mu_{x}\right)\left(h\left(x_{t}\right)-\mu_{h}\right)-E\left[\left(x_{t}-\mu_{x}\right)\left(h\left(x_{t}\right)-\mu_{h}\right)\right] \\
0
\end{array}\right]
\end{aligned}
$$


where

$$
n^{-1 / 2} \sum_{t=1}^{n}\left[\begin{array}{c}
u_{t}\left(x_{t}-\mu_{x}\right) \\
\partial g_{h}\left(x_{t}\right) / \partial x-\theta_{n l}-\left(\zeta_{x}\left(x_{t}\right)^{-1} \partial \zeta_{x}\left(x_{t}\right)\right) / \partial x u_{t}
\end{array}\right] \rightsquigarrow v(h)
$$

by Theorem 4. This follows from $\partial g_{h}\left(x_{t}\right) / \partial x=\psi_{1}+n^{-1 / 2} \partial h(x) / \partial x$ and the fact that

$$
f(y, x)=\partial g_{h}(x) / \partial x-\theta_{n l}-\left(\zeta_{x}(x)^{-1} \partial \zeta_{x}(x) / \partial x\right) u \in B_{\infty \infty}^{s}\left(\mathbb{R}^{d}, \vartheta\right)
$$

if $h(x) \in B_{\infty \infty}^{s+1}\left(\mathbb{R}^{d}, \vartheta\right)$ and $\zeta_{x}(x)^{-1} \partial \zeta_{x}(x) / \partial x \in B_{\infty \infty}^{s}\left(\mathbb{R}^{d}, \vartheta\right)$. It remains to be shown that the second term in (25) is $o_{p}(1)$. Since $\left(x_{t}-\mu_{x}\right)\left(h\left(x_{t}\right)-\mu_{h}\right) \in B_{\infty \infty}^{s+1}\left(\mathbb{R}^{d}, \vartheta-1\right)$ it follows by Nickl and Pötscher (2007, Theorem 1(2)), a strong law of large numbers for $\beta$-mixing processes and the arguments in the proof of Theorem 2.4.1. in van der Vaart and Wellner (1996, p. 122) that

$$
\sup _{h \in \mathcal{F}}\left|n^{-1} \sum_{t=1}^{n}\left(x_{t}-\mu_{x}\right)\left(h\left(x_{t}\right)-\mu_{h}\right)-E\left[\left(x_{t}-\mu_{x}\right)\left(h\left(x_{t}\right)-\mu_{h}\right)\right]\right|=o_{p}(1) .
$$

Proof of Lemma 14. The proof closely follows arguments in Newey (1994, Sections 5 and 6 ), except for the fact that $\|\cdot\|_{2, \beta}$ norms rather than Sobolev norms are the natural norms to use. This is because stochastic equicontinuity of the empirical process determining the limiting distribution is directly tied to the $\|\cdot\|_{2, \beta}$ norm. Let $\hat{m}\left(\chi_{t}, \theta, \hat{g}\right)=\hat{m}_{t}(\theta)$ and $m\left(\chi_{t}, \theta, g_{h}\right)=m_{t}(\theta)$.Consider the expansion

$$
\begin{aligned}
\sqrt{n} m_{n}\left(\theta_{0}\right) & =n^{-1 / 2} \sum_{t=1}^{n} \hat{m}_{t}\left(\theta_{0}\right)=n^{-1 / 2} \sum_{t=1}^{n}\left(m_{t}\left(\theta_{0}\right)+\gamma\left(\chi_{t}\right)\right) \\
& +n^{-1 / 2} \sum_{t=1}^{n}\left(\hat{m}_{t}\left(\theta_{0}\right)-m_{t}\left(\theta_{0}\right)-D\left(\chi_{t}, \hat{g}-g_{h}\right)\right) \\
& +n^{-1 / 2} \sum_{t=1}^{n}\left(D\left(\chi_{t}, \hat{g}-g_{h}\right)-\gamma\left(\chi_{t}\right)\right) .
\end{aligned}
$$

Let $A_{n, \varepsilon}=1\left\{\left\|n^{-1 / 2} \sum_{t=1}^{n}\left(\hat{m}_{t}\left(\theta_{0}\right)-m_{t}\left(\theta_{0}\right)+\gamma\left(\chi_{t}\right)\right)\right\|>\varepsilon\right\}$ and $B_{n, \varepsilon}=1\left\{\left\|\hat{g}-g_{h}\right\|_{2, \beta} \leq \varepsilon\right\}$. Then,

$$
\begin{aligned}
\lim _{\varepsilon \downarrow 0} \limsup _{n \rightarrow \infty} E\left[A_{n, \varepsilon}\right] & \leq \lim _{\varepsilon \downarrow 0} \limsup _{n \rightarrow \infty} E\left[A_{n, \varepsilon / 2} \cap B_{n, \varepsilon / 2}\right] \\
& +\lim _{\varepsilon \downarrow 0} \limsup _{n \rightarrow \infty} P\left(\left\|\hat{g}-g_{h}\right\|_{2, \beta}>\varepsilon / 2\right)
\end{aligned}
$$


where the second term is zero by Condition 2(ii). Consequently, all subsequent arguments are restricted to the set $B_{n, \varepsilon}$. By the Markov inequality (26) and (27) are $o_{p}(1)$ if

$$
\begin{aligned}
& E\left\|n^{-1 / 2} \sum_{t=1}^{n}\left(\hat{m}_{t}\left(\theta_{0}\right)-m_{t}\left(\theta_{0}\right)-D\left(\chi_{t}, \hat{g}-g_{h}\right)\right)\right\| \\
& \leq E\left\|n^{-1 / 2} \sum_{t=1}^{n} \hat{m}\left(\chi_{t}, \theta_{0}, \hat{g}\right)-m\left(\chi_{t}, \theta_{0}, g\right)\right\| \\
& +\sqrt{n} E\left\|m\left(\chi_{t}, \theta_{0}, \hat{g}\right)-m\left(\chi_{t}, \theta_{0}, g\right)-D\left(\chi_{t}, \hat{g}-g_{h}\right)\right\|
\end{aligned}
$$

tends to zero and

$$
\begin{aligned}
& \left\|n^{-1 / 2} \sum_{t=1}^{n}\left(D\left(\chi_{t}, \hat{g}-g_{h}\right)-\gamma\left(\chi_{t}\right)\right)\right\| \\
& \leq\left\|n^{-1 / 2} \sum_{t=1}^{n}\left(D\left(\chi_{t}, \hat{g}-g_{h}\right)-\int D\left(\chi, \hat{g}-g_{h}\right) d P\right)\right\| \\
& +\left\|\int D\left(\chi, \hat{g}-g_{h}\right) d P-n^{-1 / 2} \sum_{t=1}^{n} \gamma\left(\chi_{t}\right)\right\| \\
& =o_{p}(1) .
\end{aligned}
$$

For (28) note the first term on the RHS of the inequality is

$$
\sum_{t=1}^{n} \hat{m}\left(\chi_{t}, \theta_{0}, \hat{g}\right)-m\left(\chi_{t}, \theta_{0}, g\right)=\left[\begin{array}{c}
n\left(\bar{y}-\mu_{y}-\theta_{l}\left(\bar{x}-\mu_{x}\right)\right)\left(\bar{x}-\mu_{x}\right) \\
0
\end{array}\right] .
$$

Since

$$
\begin{aligned}
E\left\|\left(\bar{y}-\mu_{y}-\theta_{l}\left(\bar{x}-\mu_{x}\right)\right)\left(\bar{x}-\mu_{x}\right)\right\| & \leq\left(E\left\|\bar{y}-\mu_{y}-\theta_{l}\left(\bar{x}-\mu_{x}\right)\right\|^{2} E\left\|\bar{x}-\mu_{x}\right\|^{2}\right)^{1 / 2} \\
& =O\left(n^{-1}\right)
\end{aligned}
$$

it follows that the first term is $O\left(n^{-1 / 2}\right)$. For the second term in (28) note that by the same arguments as in Newey $(1994$, p. 1361) it follows that $D(\chi, g)=D(\chi) \partial g(x) / \partial x$ where $D(\chi)=\partial m\left(\chi_{t}, \theta, \phi\right) /\left.\partial \phi\right|_{\phi=\partial g(x) / \partial x}=1$. This leads to

$$
D\left(\chi, g-g_{n}\right)=\left[\begin{array}{c}
0 \\
\frac{\partial}{\partial x}\left(g-g_{h}\right)
\end{array}\right]
$$

and

$$
\left\|\left(m(\chi, \theta, g)-m\left(\chi, \theta, g_{h}\right)-D\left(\chi, g-g_{h}\right)\right)\right\|=0
$$

such that the RHS of (28) is zero and consequently, the term in (26) is $o_{p}(1)$. 
For (29) consider $D\left(\chi_{t}, g\right)=f\left(\chi_{t}\right)$ where only the second component is relevant. Thus focus on

$$
f\left(\chi_{t}\right)=\frac{\partial g\left(x_{t}\right)}{\partial x}
$$

and where $f\left(\chi_{t}\right)$ is in a class of functions indexed by $g \in \mathcal{F}_{g} \in B_{\infty \infty}^{s+1}\left(\mathbb{R}, \vartheta_{g}\right)$. It follows that $f \in \mathcal{F} \subset B_{\infty \infty}^{s}\left(\mathbb{R}, \vartheta_{g}\right)$ as long as $g \in \mathcal{F}_{g}$. By Theorem 4 the empirical process

$$
v_{n}(f):=n^{-1 / 2} \sum_{t=1}^{n}\left(f\left(\chi_{t}\right)-\int f\left(\chi_{t}\right) d P\right)
$$

satisfies $v_{n}(f) \rightsquigarrow v(f)$ where $v(f)$ is a Gaussian process. Note that Theorem 4 is established by checking all the conditions for DMR, Theorem 1. That Theorem in turn is established by establishing stochastic equicontinuity of the process $v_{n}(f)$. Now, for $f_{h, t}=\partial g_{h}\left(x_{t}\right) / \partial x$ and $f_{t}=\partial g\left(x_{t}\right) / \partial x$ it follows by from (31) that

$n^{-1 / 2} \sum_{t=1}^{n}\left(D\left(\chi_{t}, g-g_{h}\right)-\int D\left(\chi, g-g_{h}\right) d P_{0}\right)=n^{-1 / 2} \sum_{t=1}^{n}\left(f_{t}-f_{h, t}-\int\left(f_{t}-f_{h, t}\right) d P\right)$

and

$$
\begin{aligned}
& \operatorname{Pr}\left(\left\|n^{-1 / 2} \sum_{t=1}^{n}\left(D\left(\chi_{t}, \hat{g}-g_{h}\right)-\int D\left(\chi, \hat{g}-g_{h}\right) d P\right)\right\|>\delta\right) \\
& \leq \operatorname{Pr}\left(\sup _{\left\|\hat{g}-g_{h}\right\|_{2, \beta} \leq \epsilon}\left\|n^{-1 / 2} \sum_{t=1}^{n}\left(f_{t}-f_{h, t}-\int\left(f_{t}-f_{h, t}\right) d P\right)\right\|>\delta / 2\right) \\
& +\operatorname{Pr}\left(\left\|\hat{g}-g_{h}\right\|_{2, \beta}>\delta / 2\right)
\end{aligned}
$$

where (33) tends to zero as $\delta \downarrow 0$ by the fact that $v_{n}(f)$ is stochastically equicontinuous and (34) tends to zero as $\delta \downarrow 0$ by Condition 2(ii). Together (33) and (34) establishes that (29) is $o_{p}(1)$.

To establish that (30) is $o_{p}(1)$ the conditions in Newey (1994, Assumption 5.3) are sufficient: there is a function $\gamma\left(\chi_{t}\right)$ such that

$$
\begin{gathered}
E\left[\gamma\left(\chi_{t}\right)\right]=0, \\
E\left[\left\|\gamma\left(\chi_{t}\right)\right\|^{2}\right]<\infty,
\end{gathered}
$$

and for all $\left\|\hat{g}-g_{h}\right\|_{2, \beta}$ small enough,

$$
n^{-1 / 2} \sum_{t=1}^{n}\left(\gamma\left(\chi_{t}\right)-\int D\left(\chi_{t}, \hat{g}-g_{h}\right) d P\right) \rightarrow^{p} 0 .
$$


Following Newey (1994, p.1362) use 31 and integration by parts to write

$$
\begin{aligned}
E[D(\chi, g)] & =\int\left(\frac{\partial}{\partial x} g(x)\right) \zeta_{x}(x) d x=-\int\left(\partial \zeta_{x}(x) / \partial x\right) \zeta_{x}(x)^{-1} g(x) \zeta_{x}(x) d x \\
& =-E\left[\left(\partial \zeta_{x}(x) / \partial x\right) \zeta_{x}(x)^{-1} g(x)\right] .
\end{aligned}
$$

Let $\tau$ index a path (see Newey, 1994, p.1352 for a definition). Let $g\left(x_{t}, \tau\right)$ be the projection of $y_{t}$ on $\mathcal{F}$ for a path $\tau$ (see Newey, 1994, p. 1361). For $\delta(x)=\left(\partial \zeta_{x}(x) / \partial x\right) \zeta_{x}(x)^{-1}$ it follows by the projection theorem that $E_{\tau}\left[\delta\left(x_{t}\right) g\left(x_{t}, \tau\right)\right]=E_{\tau}\left[\delta\left(x_{t}\right) y_{t}\right]$. Then, Newey (1994, Eq. 4.5) implies that

$$
\partial E[D(\chi, g(\tau))] / \partial \tau=E\left[\delta\left(x_{t}\right)\left(y_{t}-g\left(x_{t}\right)\right) S\left(\chi_{t}\right)\right]
$$

where $S\left(\chi_{t}\right)$ is the score of a regular path (see Newey, 1994, Theorem 2.1). By Newey (1994, Theorem 4.1) the correction term $\gamma\left(\chi_{t}\right)$ is given by

$$
\gamma\left(\chi_{t}\right)=\delta\left(x_{t}\right) u_{t}
$$

Then $E\left[\gamma\left(\chi_{t}\right)\right]=0$ follows immediately from $E\left[u_{t} \mid x_{t}\right]=0$.

For (36) note that

$$
E\left[\left|\gamma\left(\chi_{t}\right)\right| \mid x_{t}\right] \leq \delta\left(x_{t}\right) E\left[\left|u_{t}\right| \mid x_{t}\right]
$$

Then,

$$
E\left[\left|\gamma\left(\chi_{t}\right)\right|^{2}\right] \leq E\left[\delta\left(x_{t}\right)^{2} E\left[u_{t}^{2} \mid x_{t}\right]\right] \leq E\left[\delta\left(x_{t}\right)^{2} \sigma_{t}^{2}\left(x_{t}\right)\right]<\infty
$$

where $\sigma_{t}^{2}\left(x_{t}\right)=E\left[u_{t}^{2} \mid x_{t}\right]$ and $E\left[\delta\left(x_{t}\right)^{2} \sigma_{t}^{2}\left(x_{t}\right)\right]$ is bounded by Condition (2) (i)

Finally, (37) is satisfied by Condition (2) (iii). This establishes that (26) and (27) are $o_{p}(1)$ and therefore that the first claim of the Lemma holds. The second part of the Lemma follows from Lemma (13).

Proof of Lemma 15. The estimator $\hat{\theta}_{\kappa}$ solves

$$
m_{n}\left(\hat{\theta}_{\kappa}\right)=n^{-1} \sum_{t=1}^{n} \hat{m}\left(\chi_{t}, \hat{\theta}_{\kappa}, \hat{g}_{\kappa}\right)=0
$$

which means that it can be expressed in closed form as as

$$
\left[\begin{array}{c}
\hat{\theta}_{l} \\
\hat{\theta}_{n l}
\end{array}\right]=n^{-1} \hat{Q}^{-1}\left[\begin{array}{c}
P_{1}^{\prime} M y \\
\hat{\Psi}^{\prime}\left(P^{\prime} P\right)^{-1} P^{\prime} y
\end{array}\right] .
$$

Using the fact that

$$
\left[\begin{array}{c}
\psi_{1} \\
\theta_{n l}
\end{array}\right]=n^{-1} \hat{Q}^{-1}\left[\begin{array}{c}
\psi_{1} P_{1}^{\prime} M P_{1} \\
n \theta_{n l}
\end{array}\right]
$$


it follows that

$$
\begin{aligned}
\sqrt{n}\left[\begin{array}{c}
\hat{\theta}_{l}-\psi_{1} \\
\hat{\theta}_{n l}-\theta_{n l}
\end{array}\right] & =\hat{Q}^{-1} \frac{1}{\sqrt{n}} \sum_{t=1}^{n}\left[\begin{array}{c}
\left(x_{t}-\bar{x}\right)\left(y_{t}-\psi_{1}\left(x_{t}-\bar{x}\right)\right) \\
\partial P^{\kappa}\left(x_{t}\right)^{\prime} / \partial x \hat{\psi}_{\kappa}-\theta_{n l}
\end{array}\right] \\
& =\hat{Q}^{-1} \frac{1}{\sqrt{n}} \sum_{t=1}^{n} \hat{m}_{t}\left(\chi_{t}, \theta_{0}, \hat{g}_{\kappa}\right)
\end{aligned}
$$

By Condition $3(\mathrm{i})$ it follows that $\hat{Q}^{-1}-Q^{-1}=o_{p}(1)$. Then it follows by Condition 3(ii) and (iii) that

$$
\sqrt{n}\left(\hat{\theta}_{\kappa}-\theta_{0}\right)=Q^{-1} \frac{1}{\sqrt{n}} \sum_{t=1}^{n} m_{t}\left(\chi_{t}, \theta_{0}, \hat{g}_{\kappa}\right)+o_{p}(1) .
$$

The result then follows from Lemmas 13 and 14 .

Proof of 16. It follows directly from Lemma 15 that for fixed $h$,

$$
\begin{aligned}
\tilde{H}_{1}^{1 / 2} & :=\frac{\sqrt{n}\left(\hat{\theta}_{l}-\hat{\theta}_{n l}\right)}{\sqrt{e^{\prime} \hat{Q}^{-1} \hat{\Gamma} \hat{Q}^{-1} e}}=\frac{e^{\prime}\left(\sqrt{n}\left(\hat{\theta}_{\kappa}-\theta_{0}\right)\right)+\sqrt{n} e^{\prime} \theta_{0}}{\sqrt{e^{\prime} \hat{Q^{-1}} \hat{\Gamma} \hat{Q}^{-1} e}} \\
& \rightarrow_{d} \frac{Q^{-1} v(h)}{\sqrt{e^{\prime} Q^{-1} \Gamma(h) Q^{-1} e}}+\frac{e^{\prime} Q^{-1} \tilde{b}(h)-E[\partial h(x) / \partial x]}{\sqrt{e^{\prime} Q^{-1} \Gamma(h) Q^{-1} e}}
\end{aligned}
$$

where

$$
\frac{e^{\prime} Q^{-1} \tilde{b}(h)}{\sqrt{e^{\prime} Q^{-1} \Gamma(h) Q^{-1} e}}=\frac{b(h)}{\sigma_{x}^{2} \sqrt{e^{\prime} Q^{-1} \Gamma(h) Q^{-1} e}}
$$

and

$$
\frac{Q^{-1} v(h)}{\sqrt{e^{\prime} Q^{-1} \Gamma(h) Q^{-1} e}} \sim N(0,1) .
$$

The result follows now from the continuous mapping theorem and the fact that $\tilde{H}_{1}=$ $\left(\tilde{H}_{1}^{1 / 2}\right)^{2}$. The result for $\tilde{H}_{2}$ follows in the same way. 


\section{References}

[1] Adams, R., and J. Fournier (2003): Sobolev spaces. Academic Press, New York, 2nd edn.

[2] Andrews, D. W. (1991): "An Empirical Process Central Limit Theorem for Dependent Non-Identically Distributed Random Variables," Journal of Multivariate Analysis, pp. $187-203$.

[3] Andrews, D.W. (1997): "A Conditional Kolmogorov Test," Econometrica, Vol 65, No. 5, pp.1097-1128.

[4] Andrews, D. W., and D. Pollard (1994): "An Introduction to Functional Central Limit Theorems for Dependent Stochastic Processes," International Statistical Review, 62, $119-132$.

[5] Andrews, D. W. K. (1994): "Asymptotics for Semiparametric Econometric Models via Stochastic Equicontinuity," Econometrica, 62, 43-72.

[6] Arcones, M. A., and B. Yu (1994): "Central Limit Theorems for Empirical and UProcesses of Stationary Mixing Sequences," Journal of Theoretical Probability, pp. $47-71$.

[7] Berkes, I., and W. Phillip (1977): "An almost sure invariance principle for the empirical distribution of mixing random variables," Z. Wahrscheinlichkeitstheorie und verwandte Gebiete, 41, 115-137.

[8] Bierens, H. J. (1982): "Consistent Model Specification Tests," Journal of Econometrics, Vol 20, pp. 105-134.

[9] Bierens, H. J. (1987): "A Consistent Hausman-Type Model Specification Test," Free University Research Memorandum 87-2.

[10] Bierens, H. J. and W. Ploberger (1997): "Asymptotic Theory of Integrated Conditional Moment Tests," Econometrica, Vol.65, No.5, pp. 1129-1151.

[11] Brockwell, P. J., and R. A. Davis (1991): Time Series: Theory and Methods. Springer Verlag-New York, Inc., second edn.

[12] Cox, D. R. (1961): "Tests of Separate Families of Hypotheses," Proceedings of the 4th Berkeley Symposium, 105-123. 
[13] Davidson, R. and J.G. MacKinnon (1981): "Several Tests for Model Specification in the Presence of Alternative Hypotheses," Econometrica, Vol.49, No.3, pp. 781-793.

[14] Doukhan, P., J. Leon, and F. Portal (1987): "Principe d'invariance faible pour la mesure emprique d'une suite de variables aleatoires dependantes.," Probability Theory and Related Fields, 76, 51-70.

[15] Doukhan, P., P. Massart, and E. Rio (1994): "The functional central limit theorem for strongly mixing processes," Ann. Inst. Henri Poincare Sect. B, 30, 63-82.

[16] Doukhan, P., P. Massart, and E. Rio (1995): "Invariance Principles for Absolutely Regular Empirical Processes," Annales de l'institut H. Poincare, Section B, 31, 393427.

[17] Dudley, R. (1978): "Central limit theorems for empirical measures," The Annals of Probability, 6, 899-929.

[18] Dudley, R. M. (1984): A course on Empirical Processes, Lecture Notes in Mathematics Volume 1097. Springer Verlag.

[19] Edmunds, D., and H. Triebel (1996): Function Spaces, Entropy Numbers and Differential Operators. Cambridge University Press, Cambridge.

[20] Fan, Y. and Q. Li (1996): “Consistent Model Specification Tests: Omitted Variables and Semiparametric Functional Forms," Econometrica, Vol.64, No.4, pp. 865-890.

[21] Hansen, B. E. (1996): "Stochastic Equicontinuity for Unbounded Dependent Heterogeneous Arrays," Econometric Theory, 12, 347-359.

[22] Hardle, W. and T. Stoker (1989): "Investigation of Smooth Multiple Regression by the Method of Average Derivatives," Journal of the American Statistical Association, Vol.84, pp. 986-995.

[23] Haroske, D., and H. Triebel (1994): "Entropy numbers in weighted function spaces and eigenvalue distributions of some degenerate pseudodifferential operators I," Mathematische Nachrichten, pp. 131-156.

[24] Haroske, D. D., and H. Triebel (2005): "Wavelet bases and entropy numbers in weighted function spaces," Mathematische Nachrichten, 278, 108-132. 
[25] Horowitz, J. L. and B.G. Spokoiny (2001): "An Adaptive, Rate-Optimal Test of a Parametric Mean-Regression Model Against A Nonparametric Alternative," Econometrica, Vol.69, No.3, pp. 599-631.

[26] Hausman, J. (1978): "Specification Tests in Econometrics," Econometrica, vol 46, issue $6,1251-71$.

[27] Massart, P. (1987): "Invariance Principles for Empirical Processes: the weakly dependent case. Quelques problems de vitesse de convergence pour des mesures empiriques. These d'Etat," Ph.D. thesis, Universite de Paris-Sud.

[28] Meyer, Y. (1992): Wavelets and Operators. Cambridge University Press.

[29] Newey, W. K. (1985): "Maximum Likelihood Specification Testing and Conditional Moment Tests," Econometrica, Vol 53, No. 5 pp. 1047-1070.

[30] Newey, W. K. (1994): "The Asymptotic Variance of Semiparametric Estimators," Econometrica, pp. 1349-1382.

[31] Nickl, R. (2007): "Donsker-type theorems for nonparametric maximum likelihood estimators," Probability Theory and Related Fields, 138, 411-449.

[32] Nickl, R., and B. M. Pötscher (2007): "Bracketing Metric Entropy Rates and Empirical Central Limit Theorems for Function Class of Besov and Sobolev-Type," Journal of Theoretical Probability, 20, 177-199.

[33] Ossiander, M. (1987): "A Central Limit Theorem Under Metric Entropy with $L_{2}$ Bracketing," Annals of Probability, 15, 897-919.

[34] Pollard, D. (1982): "A central limit theorems for empirical processes.," Journal of the Australian Mathematical Society, 33, 235-248.

[35] Pollard, D. (1989): "A maximal inequality for sums of independent processes under a bracketing condition," .

[36] ____ (1990): Empirical Processes: Theory and Applications, vol. 2 of CBMS Regional Conference Series in Probability and Statistics. Institute of Mathematical Statistics, Hayward, CA.

[37] Ramsey, J.B. (1969): "Tests for Specification Errors in Classical Linear Least-Squares Regression Analysis," Journal of the Royal Statistical Society. Series B, Vol 31, No. 2, pp. 350-371. 
[38] Rio, E. (1993): "Covariance Inequalities for strongly mixing processes," Annales de l'institut H. Poincare, Section B, 29(4), 587-597.

[39] ____ (1998): "Processus empiriques absolument reguliers et entropie universelle," Probability Theory and Related Fields, 111, 585-608.

[40] _-___ (2013): "Inequalities and Limit Theorems for Weakly Dependent Sequences," 3eme cycle. 2013, pp.170.

[41] Stein, E.M. (1970): Singular Integrals and Differentiability Properties of Functions. Princeton University Press, Princeton, NJ.

[42] Triebel, H. (1983): Theory of Function Spaces. Birkhäuser.

[43] van der Vaart, A. W. (1998): Asymptotic Statistics. Cambridge University Press, Cambridge.

[44] van der Vaart, A. W., and J. A. Wellner (1996): Weak Convergence and Empirical Processes. Springer Verlag.

[45] Volknoskii, V., and Y. A. Rozanov (1959): "Some Limit Theorems for Random Functions. I," Theory of Probability and its Applications, IV, 178-197.

[46] White, H. (1981): "Consequences and Detection of Misspecified Nonlinear Regression Models," Journal of the American Statistical Association, Vol. 73, No. 374, pp. 419-433.

[47] Wooldridge, J.M. (1992): "A Test for Functional Form against Nonparametric Alternatives," Econometric Theory, Vol. 8, No. 4, pp. 452-475.

[48] Yatchew, A.J. (1992): "Nonparametric Regression Tests Based on Least Squares," Econometric Theory, Vol. 8, No. 4, pp. 435-451.

[49] Zheng, J. X. (1996): "A consistent test of functional form via nonparametric estimation techniques," Journal of Econometrics, Vol. 75, pp. 263-289. 